\title{
Gravitational spin-orbit dynamics at the fifth-and-a-half post-Newtonian order
}

\author{
Mohammed Khalilø* \\ Max Planck Institute for Gravitational Physics (Albert Einstein Institute), \\ Am Mühlenberg 1, Potsdam 14476, Germany \\ and Department of Physics, University of Maryland, College Park, Maryland 20742, USA
}

(Received 27 October 2021; accepted 3 November 2021; published 3 December 2021)

\begin{abstract}
Accurate waveform models are crucial for gravitational-wave data analysis, and since spin has a significant effect on the binary dynamics, it is important to improve the spin description in these models. In this paper, we derive the spin-orbit (SO) coupling at the fifth-and-a-half post-Newtonian (5.5PN) order. The method we use splits the conservative dynamics into local and nonlocal-in-time parts, and then relates the local-in-time part to gravitational self-force results by exploiting the simple mass-ratio dependence of the post-Minkowskian expansion of the scattering angle. We calculate the nonlocal contribution to the 5.5PN SO dynamics to eighth order in the small-eccentricity expansion for bound orbits, and to leading order in the large-eccentricity expansion for unbound orbits. For the local contribution, we obtain all of the 5.5PN SO coefficients from first-order self-force results for the redshift and spin-precession invariants, except for one unknown that could be fixed in the future by second-order self-force results. However, by incorporating our 5.5PN results in the effective-one-body formalism and comparing its binding energy to numerical relativity, we find that the remaining unknown has a small effect on the SO dynamics, demonstrating an improvement in accuracy at that order.
\end{abstract}

DOI: $10.1103 /$ PhysRevD.104.124015

\section{INTRODUCTION}

Gravitational-wave (GW) observations [1-3] have improved our understanding of compact binary systems, their properties, and their formation channels [4,5]. A crucial component in searching for GW signals and inferring their parameters is accurate analytical waveform models, in which spin is an important ingredient given its significant effect on the orbital dynamics.

Three main analytical approximation methods exist for describing the dynamics during the inspiral phase: the post-Newtonian (PN), the post-Minkowskian (PM), and the small-mass-ratio [gravitational self-force (GSF)] approximations.

The PN approximation is valid for small velocities and weak gravitational potential $v^{2} / c^{2} \sim G M / r c^{2} \ll 1$, and is most applicable for comparable-mass binaries in bound orbits. Many studies have contributed to improving the description of the conservative PN dynamics, for

*mohammed.khalil@aei.mpg.de

Published by the American Physical Society under the terms of the Creative Commons Attribution 4.0 International license. Further distribution of this work must maintain attribution to the author(s) and the published article's title, journal citation, and DOI. Open access publication funded by the Max Planck Society. nonspinning binaries [6-20], at the spin-orbit (SO) level [21-35], spin-spin [36-44], and higher orders in spin [45-50]. For reviews, see Refs. [51-56].

The PM approximation is valid for arbitrary velocities in the weak field $G M / r c^{2} \ll 1$, and is most applicable for scattering motion since relativistic velocities can be achieved. It was pioneered by the classic results of Westpfahl [57,58], with rapid progress using classical methods [59-65], scattering amplitudes [66-75], effective field theory [76-80], and worldline quantum field theory $[81,82]$. Spin effects were included in PM expansions using all of these approaches in Refs. [83-96], and radiative contributions in Refs. [97-101].

The small-mass-ratio approximation $m_{1} / m_{2} \ll 1$ is based on GSF theory, and is most applicable for extreme-massratio inspirals (see, e.g., Refs. [102-122] and the reviews [123-126].) Analytic GSF calculations to high PN orders were performed at first order in the mass ratio for the gaugeinvariant redshift [127-135] and the spin-precession frequency [136-141]. There has also been recent important work on numerically calculating the binding energy and energy flux at second order in the mass ratio [142,143].

The effective-one-body (EOB) formalism [144,145] combines information from different analytical approximations with numerical relativity (NR) results, while recovering the strong-field test-body limit, thereby extending each approximation's domain of validity and improving 
the inspiral-merger-ringdown waveforms. EOB models have been constructed for nonspinning [10,146-150], spinning [151-166], and eccentric binaries [167-170]. In addition, information from the post-Minkowskian $[62,63,171,172]$ and small mass-ratio approximations [173-176] have been incorporated in EOB models.

Recently, a method [177] (sometimes dubbed the "Tutti Frutti" method [98]) that combines all of these formalisms has been used to derive PN results valid for arbitrary mass ratios from GSF results at first order in the mass ratio. The method relies on the simple mass-ratio dependence of the PM-expanded scattering angle [64] (see also Ref. [86]), making it possible to relate the local-in-time part of the Hamiltonian, or radial action, to GSF invariants, such as the redshift and precession frequency. The nonlocal-in-time part of the conservative dynamics, due to backscattered radiation emitted at earlier times, is derived separately, since it is calculated in an eccentricity expansion that differs between bound and unbound orbits. This approach has been used to derive the 5PN conservative dynamics for nonspinning binaries except for two coefficients [177,178], the 6PN dynamics except for four coefficients $[179,180]$, and the full 4.5PN SO and 5PN aligned $\operatorname{spin}_{1}-\operatorname{spin}_{2}$ dynamics [181,182].

In this paper, we determine the 5.5PN SO coupling for the two-body dynamics, which is the fourth-subleading PN order, except for one coefficient at second order in the mass ratio. Throughout, we perform all calculations for spins aligned, or antialigned, with the direction of the orbital angular momentum. However, the results are valid for precessing spins [181], since at the SO level the spin vector only couples to the angular momentum vector.

The results of this paper and the procedure used can be summarized as follows:

(1) In Sec. II, we calculate the nonlocal contribution to the 5.5PN SO Hamiltonian for bound orbits, in a small-eccentricity expansion up to eighth order in eccentricity. We do this for a harmonic-coordinates Hamiltonian, and then incorporate those results into the gyro-gravitomagnetic factors in an EOB Hamiltonian.

(2) In Sec. III, we determine the local contribution by relating the coefficients of the local Hamiltonian to those of the PM-expanded scattering angle. We then calculate the redshift and spin-precession invariants from the total Hamiltonian, and match their smallmass-ratio expansion to first-order self-force (1SF) results. This allows us to recover all of the coefficients of the local part except for one unknown. However, by computing the EOB binding energy and comparing it to NR, we show that the effect of the remaining unknown on the dynamics is small.

(3) In Sec. IV, we complement our results for unbound orbits by calculating the nonlocal part of the gaugeinvariant scattering angle, to leading order in the large-eccentricity expansion.
(4) In Sec. V, we provide two gauge-invariant quantities that characterize bound orbits: the radial action as a function of energy and angular momentum, and the circular-orbit binding energy as a function of frequency.

We conclude in Sec. VI with a discussion of the results, and in the Appendix we provide a summary of the quasiKeplerian parametrization at leading SO order. The main results of this paper are provided in the Supplemental Material as a Mathematica file [183].

\section{A. Notation}

We use the metric signature $(-,+,+,+)$, and units in which $G=c=1$, but sometimes write them explicitly in $\mathrm{PM}$ and PN expansions for clarity.

For a binary with masses $m_{1}$ and $m_{2}$, with $m_{2} \geq m_{1}$, and spins $\boldsymbol{S}_{1}$ and $\boldsymbol{S}_{2}$, we define the following combinations of the masses:

$$
\begin{aligned}
M & =m_{1}+m_{2}, \quad \mu=\frac{m_{1} m_{2}}{M}, \quad \nu=\frac{\mu}{M}, \\
q & =\frac{m_{1}}{m_{2}}, \quad \delta=\frac{m_{2}-m_{1}}{M} .
\end{aligned}
$$

We define the mass-rescaled spins

$$
\boldsymbol{a}_{1}=\frac{\boldsymbol{S}_{1}}{m_{1}}, \quad \boldsymbol{a}_{2}=\frac{\boldsymbol{S}_{2}}{m_{2}},
$$

the dimensionless spin magnitudes

$$
\chi_{1}=\frac{\left|S_{1}\right|}{m_{1}^{2}}, \quad \chi_{2}=\frac{\left|S_{2}\right|}{m_{2}^{2}},
$$

and the spin combinations

$$
\begin{gathered}
\boldsymbol{S}=\boldsymbol{S}_{1}+\boldsymbol{S}_{2}, \quad \boldsymbol{S}^{*}=\frac{m_{2}}{m_{1}} \boldsymbol{S}_{1}+\frac{m_{1}}{m_{2}} \boldsymbol{S}_{2}, \\
\chi_{S}=\frac{1}{2}\left(\chi_{1}+\chi_{2}\right), \quad \chi_{A}=\frac{1}{2}\left(\chi_{2}-\chi_{1}\right) .
\end{gathered}
$$

We use several variables related to the total energy $E$ of the binary system: the binding energy $\bar{E}=E-M c^{2}$, the mass-rescaled energy $\Gamma=E / M$, and the effective energy $E_{\text {eff }}$ defined by the energy map

$$
E=M \sqrt{1+2 \nu\left(\frac{E_{\mathrm{eff}}}{\mu}-1\right)} .
$$

We also define the asymptotic relative velocity $v$ and Lorentz factor $\gamma$ via

$\gamma=\frac{E_{\mathrm{eff}}}{\mu}, \quad v=\frac{\sqrt{\gamma^{2}-1}}{\gamma}, \quad \leftrightarrow \quad \gamma=\frac{1}{\sqrt{1-v^{2}}}$, 
and define the dimensionless energy variable

$$
\varepsilon=\gamma^{2}-1=\gamma^{2} v^{2},
$$

(note that $\varepsilon$ used here is denoted as $p_{\infty}^{2}$ in Ref. [178]).

The magnitude of the orbital angular momentum is denoted by $L$, and is related to the relative position $r$, radial momentum $p_{r}$, and total linear momentum $p$ via

$$
p^{2}=p_{r}^{2}+\frac{L^{2}}{r^{2}} .
$$

We often use dimensionless rescaled quantities, such as

$$
\begin{aligned}
r=\frac{r^{\text {phys }}}{M}, & L=\frac{L^{\text {phys }}}{M \mu}, \quad p_{r}=\frac{p_{r}^{\text {phys }}}{\mu}, \\
E=\frac{E^{\text {phys }}}{\mu}, & H=\frac{H^{\text {phys }}}{\mu}, \\
&
\end{aligned}
$$

and similarly for related variables, e.g., $\bar{E}=\bar{E}^{\text {phys }} / \mu$, etc. It should be clear from the context whether physical or rescaled quantities are being used.

\section{NONLOCAL 5.5PN SO HAMILTONIAN FOR BOUND ORBITS}

The total conservative action at a given PN order can be split into local and nonlocal-in-time parts, such that

$$
S_{\mathrm{tot}}^{\mathrm{nPN}}=S_{\mathrm{loc}}^{\mathrm{nPN}}+S_{\mathrm{nonloc}}^{\mathrm{nPN}}
$$

where the nonlocal part is due to tail effects projected on the conservative dynamics $[9,184,185]$, i.e., radiation emitted at earlier times and backscattered onto the binary. The nonlocal contribution starts at 4PN order, and has been derived for nonspinning binaries up to 6PN order $[10,178,179]$. In this section, we derive the leading-order spin contribution to the nonlocal part, which is at 5.5PN order.

The nonlocal part of the action can be calculated via the following integral:

$$
S_{\text {nonloc }}^{\mathrm{LO}}=\frac{G M}{c^{3}} \int d t \mathrm{Pf}_{2 s / c} \int \frac{d t^{\prime}}{\left|t-t^{\prime}\right|} \mathcal{F}_{\mathrm{LO}}^{\text {split }}\left(t, t^{\prime}\right),
$$

where the label "LO" here means that we include the leading-order nonspinning and SO contributions, and where the Hadamard partie finie (Pf) operation is used since the integral is logarithmically divergent at $t^{\prime}=t$. The time-split (or time-symmetric) GW energy flux $\mathcal{F}_{\mathrm{LO}}^{\text {split }}\left(t, t^{\prime}\right)$ is written in terms of the source multipole moments as [9]

$$
\mathcal{F}_{\mathrm{LO}}^{\text {split }}\left(t, t^{\prime}\right)=\frac{G}{c^{5}}\left[\frac{1}{5} I_{i j}^{(3)}(t) I_{i j}^{(3)}\left(t^{\prime}\right)+\frac{16}{45 c^{2}} J_{i j}^{(3)}(t) J_{i j}^{(3)}\left(t^{\prime}\right)\right] .
$$

The mass quadrupole $I^{i j}$ and the current quadrupole $J^{i j}$ (in harmonic coordinates and using the Newton-Wigner spinsupplementary condition $[186,187])$ are given by $[188,189]$

$$
\begin{aligned}
I_{i j}= & m_{1} x_{1}^{\langle i} x_{1}^{j\rangle}+\frac{3}{c^{3}} x_{1}^{\langle i}\left(\boldsymbol{v}_{1} \times \boldsymbol{S}_{1}\right)^{j\rangle} \\
& -\frac{4}{3 c^{3}} \frac{d}{d t} x_{1}^{\langle i}\left(\boldsymbol{x}_{1} \times \boldsymbol{S}_{1}\right)^{j\rangle}+1 \leftrightarrow 2, \\
J_{i j}= & m_{1} x_{1}^{\langle i}\left(\boldsymbol{x}_{1} \times \boldsymbol{v}_{1}\right)^{j\rangle}+\frac{3}{2 c} x_{1}^{\langle i} S_{1}^{j\rangle}+1 \leftrightarrow 2,
\end{aligned}
$$

where the indices in angle brackets denote a symmetric trace-free part.

As was shown in Refs. $[9,10]$, the nonlocal part of the action can be written in terms of $\tau \equiv t^{\prime}-t$ as

$$
\begin{aligned}
S_{\text {nonloc }}^{\mathrm{LO}}= & -\int d t \delta H_{\text {nonloc }}^{\mathrm{LO}}(t), \\
\delta H_{\text {nonloc }}^{\mathrm{LO}}(t)= & -\frac{G M}{c^{3}} \operatorname{Pf}_{2 s / c} \int \frac{d \tau}{|\tau|} \mathcal{F}_{\mathrm{LO}}^{\text {split }}(t, t+\tau) \\
& +2 \frac{G M}{c^{3}} \mathcal{F}_{\mathrm{LO}}^{\mathrm{split}}(t, t) \ln \left(\frac{r}{s}\right) .
\end{aligned}
$$

Following Ref. [178], we choose the arbitrary length scale $s$ entering the partie finie operation to be the radial distance $r$ between the two bodies in harmonic coordinates. This has the advantage of simplifying the local part by removing its dependence on $\ln r$.

\section{A. Computation of the nonlocal Hamiltonian in a small-eccentricity expansion}

The integral for the nonlocal Hamiltonian in Eq. (2.5) can be performed in a small-eccentricity expansion using the quasi-Keplerian parametrization [190], which can be expressed, up to $1.5 \mathrm{PN}$ order, by the following equations:

$$
\begin{gathered}
r=a_{r}\left(1-e_{r} \cos u\right), \\
\ell=n t=u-e_{t} \sin u, \\
\phi=2 K \arctan \left[\sqrt{\frac{1+e_{\phi}}{1-e_{\phi}}} \tan \frac{u}{2}\right],
\end{gathered}
$$

where $a_{r}$ is the semimajor axis, $u$ is the eccentric anomaly, $\ell$ is the mean anomaly, $n$ is the mean motion (radial angular frequency), $K$ is the periastron advance, and $\left(e_{r}, e_{t}, e_{\phi}\right)$ are the radial, time, and phase eccentricities.

The quasi-Keplerian parametrization was generalized to 3PN order in Ref. [191], and including SO and spinspin contributions in Refs. [192,193]. In Appendix A 1 we summarize the relations between the quantities used in the quasi-Keplerian parametrization and the energy and angular momentum at leading $\mathrm{SO}$ order. Using the 
quasi-Keplerian parametrization, we express the source multipole moments in terms of the variables $\left(a_{r}, e_{t}, t\right)$, and expand the moments in eccentricity.

In the center-of-mass frame, the position vectors of the two bodies, $x_{1}$ and $x_{2}$, are related to $\boldsymbol{x} \equiv \boldsymbol{x}_{1}-\boldsymbol{x}_{2}$ via [194]

$$
\begin{aligned}
x_{1}^{i} & =\frac{m_{2}}{M} x^{i}-\frac{\nu}{2 c^{3} \delta M} \epsilon_{j k}^{i} v^{j}\left(S^{k}-S^{* k}\right), \\
x_{2}^{i} & =-\frac{m_{1}}{M} x^{i}-\frac{\nu}{2 c^{3} \delta M} \epsilon_{j k}^{i} v^{j}\left(S^{k}-S^{* k}\right),
\end{aligned}
$$

where $\boldsymbol{v} \equiv \boldsymbol{v}_{1}-\boldsymbol{v}_{2}$, and hence the source moments from Eq. (2.4) can be written as

$$
\begin{aligned}
I_{i j}= & M \nu x^{\langle i} x^{j\rangle}+\frac{1}{3 c^{3}}\left[\frac { m _ { 2 } ^ { 2 } } { M ^ { 2 } } \left(4 v^{\langle i}\left(\boldsymbol{S}_{1} \times \boldsymbol{x}\right)^{j\rangle}\right.\right. \\
& \left.\left.-5 x^{\langle i}\left(\boldsymbol{S}_{1} \times \boldsymbol{v}\right)^{j\rangle}\right)+1 \leftrightarrow 2\right], \\
J_{i j}= & M \delta \nu x^{\langle i}(\boldsymbol{v} \times \boldsymbol{x})^{j\rangle}+\frac{3}{2 c}\left[\frac{m_{2}}{M} x^{\langle i} S_{1}^{j\rangle}-\frac{m_{1}}{M} x^{\langle i} S_{2}^{j\rangle}\right] .
\end{aligned}
$$

In polar coordinates,

$$
\begin{aligned}
& \boldsymbol{x}=r(\cos \phi, \sin \phi), \\
& \boldsymbol{v}=\dot{r}(\cos \phi, \sin \phi)+r \dot{\phi}(-\sin \phi, \cos \phi),
\end{aligned}
$$

with $r$ and $\phi$ given by Eqs. (2.6) and (2.8), $e_{r}$ and $e_{\phi}$ related to $e_{t}$ via Eqs. (A12) and (A13), and $\dot{r}$ and $\dot{\phi}$ are given by

$$
\begin{gathered}
\dot{r}=\frac{e_{t} \sin u}{\sqrt{a_{r}}\left(1-e_{t} \cos u\right)}, \\
\dot{\phi}=\frac{\sqrt{a_{r}-a_{r} e_{t}^{2}}}{a_{r}^{2}\left(1-e_{t} \cos u\right)^{2}},
\end{gathered}
$$

which are only needed at leading order. We then write the eccentric anomaly $u$ in terms of time $t$ using Kepler's equation (2.7), which has a solution in terms of a FourierBessel series expansion,

$$
\begin{aligned}
u= & n t+\sum_{k=1}^{\infty} \frac{2}{k} J_{k}\left(k e_{t}\right) \sin (k n t) \\
& \simeq n t+e_{t} \sin (n t)+\frac{1}{2} e_{t}^{2} \sin (2 n t)+\ldots
\end{aligned}
$$

We perform the eccentricity expansion for the nonlocal part up to $\mathcal{O}\left(e_{t}^{8}\right)$ since it corresponds to an expansion to $\mathcal{O}\left(p_{r}^{8}\right)$, which is the highest power of $p_{r}$ in the 5.5PN SO local part. However, to simplify the presentation, we write the intermediate steps only expanded to $\mathcal{O}\left(e_{t}\right)$.

Plugging the expressions for $(r, \phi, \dot{r}, \dot{\phi})$ in terms of $\left(a_{r}, e_{t}, t\right)$ into the source moments used in the time-split energy flux (2.3) and expanding in eccentricity yields

$$
\begin{aligned}
\mathcal{F}_{\mathrm{LO}}^{\text {split }}(t, t+\tau)= & \nu^{2} a_{r}^{4} n^{6}\left\{\frac{32}{5} \cos (2 n \tau)+\frac{12}{5} e_{t}[9 \cos (n t+3 n \tau)+9 \cos (n t-2 n \tau)-\cos (n t-n \tau)-\cos (n t+2 n \tau)]\right\} \\
& +\frac{8 \nu^{2}}{15} n^{6} a_{r}^{5 / 2}\left\{48 n \tau \sin (2 n \tau)\left(2 \delta \chi_{A}-\nu \chi_{S}+2 \chi_{S}\right)-32 \cos (2 n \tau)\left(9 \delta \chi_{A}-5 \nu \chi_{S}+9 \chi_{S}\right)\right. \\
& -\cos (n \tau)\left(\delta \chi_{A}-4 \nu \chi_{S}+\chi_{S}\right)+e_{t} \cos \left(n t+\frac{n \tau}{2}\right)\left[\cos \left(\frac{3 n \tau}{2}\right)\left(352 \delta \chi_{A}+352 \chi_{S}-157 \nu \chi_{S}\right)\right. \\
& \left.\left.-27 \cos \left(\frac{5 n \tau}{2}\right)\left(84 \delta \chi_{A}-47 \nu \chi_{S}+84 \chi_{S}\right)-36 n \tau\left(\sin \left(\frac{3 n \tau}{2}\right)-9 \sin \left(\frac{5 n \tau}{2}\right)\right)\left(2 \delta \chi_{A}-\nu \chi_{S}+2 \chi_{S}\right)\right]\right\} \\
& +\mathcal{O}\left(e_{t}^{2}\right),
\end{aligned}
$$

the orbit average of which is given by

$$
\begin{aligned}
\left\langle\mathcal{F}_{\mathrm{LO}}^{\text {split }}(t, t+\tau)\right\rangle= & \frac{n}{2 \pi} \int_{0}^{2 \pi / n} \mathcal{F}_{\mathrm{LO}}^{\text {split }}(t, t+\tau) d t \\
= & \frac{32}{5} \nu^{2} n^{6} a_{r}^{4} \cos (2 n \tau)+\frac{8}{15} \nu^{2} n^{6} a_{r}^{5 / 2}\left[48 n \tau \sin (2 n \tau)\left(2 \delta \chi_{A}-\nu \chi_{S}+2 \chi_{S}\right)-\cos (n \tau)\left(\delta \chi_{A}-4 \nu \chi_{S}+\chi_{S}\right)\right. \\
& \left.-32 \cos (2 n \tau)\left(9 \delta \chi_{A}-5 \nu \chi_{S}+9 \chi_{S}\right)\right]+\mathcal{O}\left(e_{t}^{2}\right)
\end{aligned}
$$

In the limit $\tau=0$, this equation agrees with the eccentricity expansion of the energy flux from Eq. (64) of Ref. [193].

Then, we perform the partie finie operation with time scale $2 s / c$ using Eq. (4.2) of Ref. [9], which reads

$$
\operatorname{Pf}_{T} \int_{0}^{\infty} \frac{d v}{v} g(v)=\int_{0}^{T} \frac{d v}{v}[g(v)-g(0)]+\int_{T}^{\infty} \frac{d v}{v} g(v) .
$$


The first line of Eq. (2.5) yields

$$
\begin{aligned}
& -\mathrm{Pf}_{2 s / c} \int \frac{d \tau}{|\tau|}\left\langle\mathcal{F}_{\mathrm{LO}}^{\mathrm{split}}(t, t+\tau)\right\rangle \\
& =\frac{64}{5} \nu^{2} n^{6} a_{r}^{4}\left[\ln (4 n s)+\gamma_{E}\right]-\frac{16}{15} \nu^{2} n^{6} a_{r}^{5 / 2}\left\{\left[289 \delta \chi_{A}+(289-164 \nu) \chi_{S}\right] \ln (n s)\right. \\
& \left.\quad+\left[289 \gamma_{E}+48+577 \ln 2\right] \delta \chi_{A}+\left[\gamma_{E}(289-164 \nu)-12 \nu(2+27 \ln 2)+48+577 \ln 2\right] \chi_{S}\right\}+\mathcal{O}\left(e_{t}^{2}\right),
\end{aligned}
$$

while the second line yields

$$
2\left\langle\mathcal{F}_{\mathrm{LO}}^{\text {split }}(t, t)\right\rangle \ln \left(\frac{r}{s}\right)=\frac{64}{5} \nu^{2} n^{6} a_{r}^{4} \ln \left(\frac{a_{r}}{s}\right)-\frac{16}{15} \nu^{2} n^{6} a_{r}^{5 / 2} \ln \left(\frac{a_{r}}{s}\right)\left[289 \delta \chi_{A}+(289-164 \nu) \chi_{S}\right]+\mathcal{O}\left(e_{t}^{2}\right) .
$$

Adding the two expressions removes the dependence on $s$.

When performing the calculation to $\mathcal{O}\left(e_{t}^{8}\right)$, we obtain the following Delaunay-averaged nonlocal Hamiltonian:

$$
\begin{aligned}
& \left\langle\delta H_{\text {nonloc }}^{\mathrm{LO}}\right\rangle=\frac{\nu^{2}}{a_{r}^{5}}\left[\mathcal{A}^{4 \mathrm{PN}}\left(e_{t}\right)+\mathcal{B}^{4 \mathrm{PN}}\left(e_{t}\right) \ln a_{r}\right]+\frac{\nu^{2} \delta \chi_{A}}{a_{r}^{13 / 2}}\left\{\frac{584}{15} \ln a_{r}-\frac{64}{5}-\frac{464}{3} \ln 2-\frac{1168}{15} \gamma_{E}\right. \\
& +e_{t}^{2}\left[\frac{2908}{5} \ln a_{r}-\frac{5816}{5} \gamma_{E}+\frac{2172}{5}-\frac{3304}{15} \ln 2-\frac{10206}{5} \ln 3\right] \\
& +e_{t}^{4}\left[\frac{43843}{15} \ln a_{r}-\frac{87686}{15} \gamma_{E}+\frac{114991}{30}-\frac{201362}{5} \ln 2+\frac{48843}{4} \ln 3\right] \\
& +e_{t}^{6}\left[\frac{55313}{6} \ln a_{r}-\frac{55313}{3} \gamma_{E}+\frac{961807}{60}+\frac{6896921}{45} \ln 2-\frac{3236031}{160} \ln 3-\frac{24296875}{288} \ln 5\right] \\
& \left.+e_{t}^{8}\left[\frac{134921}{6} \ln a_{r}-\frac{134921}{3} \gamma_{E}+\frac{135264629}{2880}-\frac{94244416}{135} \ln 2+\frac{12145234375}{27648} \ln 5-\frac{1684497627}{5120} \ln 3\right]\right\} \\
& +\frac{\nu^{2} \chi_{S}}{a_{r}^{13 / 2}}\left\{-\frac{64}{5}+\frac{32 \nu}{5}+\left(\frac{896 \nu}{15}-\frac{1168}{15}\right) \gamma_{E}+\left(\frac{576 \nu}{5}-\frac{464}{3}\right) \ln 2+\left(\frac{584}{15}-\frac{448 \nu}{15}\right) \ln a_{r}\right. \\
& +e_{t}^{2}\left[\frac{2172}{5}-\frac{4412 \nu}{15}+\left(\frac{4216 \nu}{5}-\frac{5816}{5}\right) \gamma_{E}+\left(\frac{5192 \nu}{15}-\frac{3304}{15}\right) \ln 2+\left(\frac{6561 \nu}{5}-\frac{10206}{5}\right) \ln 3\right. \\
& \left.+\left(\frac{2908}{5}-\frac{2108 \nu}{5}\right) \ln a_{r}\right]+e_{t}^{4}\left[\frac{114991}{30}-\frac{38702 \nu}{15}+\left(\frac{62134 \nu}{15}-\frac{87686}{15}\right) \gamma_{E}\right. \\
& \left.+\left(\frac{386414 \nu}{15}-\frac{201362}{5}\right) \ln 2+\left(\frac{48843}{4}-\frac{28431 \nu}{4}\right) \ln 3+\left(\frac{43843}{15}-\frac{31067 \nu}{15}\right) \ln a_{r}\right] \\
& +e_{t}^{6}\left[\frac{961807}{60}-\frac{215703 \nu}{20}+\left(\frac{193718 \nu}{15}-\frac{55313}{3}\right) \gamma_{E}+\left(\frac{6896921}{45}-\frac{12343118 \nu}{135}\right) \ln 2\right. \\
& \left.+\left(\frac{3768201 \nu}{320}-\frac{3236031}{160}\right) \ln 3+\left(\frac{92421875 \nu}{1728}-\frac{24296875}{288}\right) \ln 5+\left(\frac{55313}{6}-\frac{96859 \nu}{15}\right) \ln a_{r}\right] \\
& +e_{t}^{8}\left[\frac{135264629}{2880}-\frac{45491177 \nu}{1440}+\left(\frac{93850 \nu}{3}-\frac{134921}{3}\right) \gamma_{E}+\left(\frac{118966123 \nu}{270}-\frac{94244416}{135}\right) \ln 2\right. \\
& +\left(\frac{537837489 \nu}{2560}-\frac{1684497627}{5120}\right) \ln 3+\left(\frac{12145234375}{27648}-\frac{3790703125 \nu}{13824}\right) \ln 5 \\
& \left.\left.+\left(\frac{134921}{6}-\frac{46925 \nu}{3}\right) \ln a_{r}\right]\right\}+\mathcal{O}\left(e_{t}^{10}\right),
\end{aligned}
$$

where the functions $\mathcal{A}^{4 \mathrm{PN}}\left(e_{t}\right)$ and $\mathcal{B}^{4 \mathrm{PN}}\left(e_{t}\right)$ in the 4PN part are given in Table I of Ref. [178]. 


\section{B. Nonlocal part of the EOB Hamiltonian}

The (dimensionless) EOB Hamiltonian is given by the energy map

$$
H_{\mathrm{EOB}}=\frac{1}{\nu} \sqrt{1+2 \nu\left(H_{\mathrm{eff}}-1\right)},
$$

where the effective Hamiltonian is

$$
\begin{aligned}
H_{\mathrm{eff}}= & \sqrt{A(r)\left[1+p^{2}+(A(r) \bar{D}(r)-1) p_{r}^{2}+Q\left(r, p_{r}\right)\right]} \\
& +\frac{1}{c^{3} r^{3}} \boldsymbol{L} \cdot\left[g_{S}\left(r, p_{r}\right) \boldsymbol{S}+g_{S^{*}}\left(r, p_{r}\right) \boldsymbol{S}^{*}\right]
\end{aligned}
$$

The nonspinning potentials $A, \bar{D}$, and $Q$ were obtained at 4PN order in Ref. [10]. The 4.5PN gyro-gravitomagnetic factors, $g_{S}$ and $g_{S^{*}}$, are given by Eq. (5.6) of Ref. [182], and are in a gauge such that they are independent of the angular momentum. Note that the gyro-gravitomagnetic factors are the same for both aligned and precessing spins, since the spin vector only couples to the angular momentum vector at the SO level. Hence, even though the calculations are specialized to aligned spins, the final result for the gyro-gravitomagnetic factors is valid for precessing spins.

Splitting the potentials $A, \bar{D}, Q$ into a local and a nonlocal piece, and writing the gyro-gravitomagnetic factors as

$$
\begin{gathered}
g_{S}=2+\cdots+\frac{1}{c^{8}}\left(g_{S}^{5.5 \mathrm{PN}, \mathrm{loc}}+g_{S}^{5.5 \mathrm{PN}, \mathrm{nonloc}}\right), \\
g_{S^{*}}=\frac{3}{2}+\cdots+\frac{1}{c^{8}}\left(g_{S^{*}}^{5.5 \mathrm{PN}, \mathrm{loc}}+g_{S^{*}}^{5.5 \mathrm{PN}, \text { nonloc }}\right)
\end{gathered}
$$

yields the following LO nonlocal part of the PN-expanded effective Hamiltonian:

$$
\begin{aligned}
H_{\mathrm{eff}}= & H_{\mathrm{eff}}^{\mathrm{loc}}+\frac{1}{c^{8}} H_{\mathrm{eff}}^{\mathrm{nonloc}}+\mathcal{O}(5 \mathrm{PN}, 6.5 \mathrm{PNSO}), \\
H_{\mathrm{eff}}^{\text {nonloc }}= & \frac{1}{2}\left(A^{\text {nonloc }}+\bar{D}^{\text {nonloc }} p_{r}^{2}+Q^{\text {nonloc }}\right) \\
& +\frac{\nu \boldsymbol{L}}{c^{3} r^{3}} \cdot\left[S g_{S}^{5.5 \mathrm{PN}, \text { nonloc }}+S^{*} g_{S^{*}}^{5.5 \mathrm{PN}, \text { nonloc }}\right] .
\end{aligned}
$$

Then, we write the nonlocal piece of the potentials and gyro-gravitomagnetic factors in terms of unknown coefficients, calculate the Delaunay average of $H_{\text {eff }}^{\text {non }}$ in terms of the EOB coordinates $\left(a_{r}, e_{t}\right)$, and match it to the harmoniccoordinates Hamiltonian from Eq. (2.19). Since harmonic and EOB coordinates agree at leading SO order, no canonical transformation is needed between the two at that order.

This yields the results in Table IV of Ref. [178] for the $4 \mathrm{PN}$ nonspinning part, and the following SO part expanded to $\mathcal{O}\left(p_{r}^{8}\right)$ :

$$
\begin{aligned}
g_{S}^{5.5 P N, \text { nonloc }} & =2 \nu\left\{\left(\frac{292}{15} \ln r-\frac{32}{5}-\frac{584}{15} \gamma_{E}-\frac{232}{3} \ln 2\right) \frac{1}{r^{4}}+\left(\frac{12782}{15}-104 \gamma_{E}+\frac{32744}{15} \ln 2-\frac{11664}{5} \ln 3+52 \ln r\right) \frac{p_{r}^{2}}{r^{3}}\right. \\
& +\left(\frac{12503}{15}-\frac{635456}{9} \ln 2+\frac{218943}{5} \ln 3\right) \frac{p_{r}^{4}}{r^{2}}+\left(\frac{38246}{25}+\frac{176799232}{225} \ln 2-\frac{2517237}{10} \ln 3-\frac{3015625}{18} \ln 5\right) \frac{p_{r}^{6}}{r} \\
& \left.+\left(\frac{503099}{350}-\frac{898982848}{189} \ln 2+\frac{6352671875}{3024} \ln 5-\frac{31129029}{400} \ln 3\right) p_{r}^{8}+\mathcal{O}\left(p_{r}^{10}\right)\right\}, \\
g_{S^{*}}^{5.5 \text { PN,nonloc }} & 3 \\
= & \frac{3}{2} \nu\left\{\left(16 \ln r-\frac{32}{5}-32 \gamma_{E}-\frac{2912}{45} \ln 2\right) \frac{1}{r^{4}}+\left(\frac{35024}{45}-\frac{1024 \gamma_{E}}{15}+\frac{93952}{45} \ln 2-\frac{10692}{5} \ln 3+\frac{512}{15} \ln r\right) \frac{p_{r}^{2}}{r^{3}}\right. \\
& +\left(\frac{9232}{15}-\frac{2978624}{45} \ln 2+\frac{206064}{5} \ln 3\right) \frac{p_{r}^{4}}{r^{2}}+\left(\frac{33048}{25}+\frac{1497436672}{2025} \ln 2-\frac{1199934}{5} \ln 3-\frac{12593750}{81} \ln 5\right) \frac{p_{r}^{6}}{r} \\
& \left.+\left(\frac{651176}{525}-\frac{9076395968}{2025} \ln 2+\frac{2226734375}{1134} \ln 5-\frac{697653}{14} \ln 3\right) p_{r}^{8}+\mathcal{O}\left(p_{r}^{10}\right)\right\} .
\end{aligned}
$$

\section{LOCAL 5.5PN SO HAMILTONIAN AND SCATTERING ANGLE}

In this section, we determine the local part of the Hamiltonian and scattering angle from $1 \mathrm{SF}$ results by making use of the simple mass dependence of the PMexpanded scattering angle.

\section{A. Mass dependence of the scattering angle}

Based on the structure of the PM expansion, Poincaré symmetry, and dimensional analysis, Ref. [64] (see also Ref. [86]) showed that the magnitude of the impulse (net change in momentum), for nonspinning systems in the center-of-mass frame, has the following dependence on the masses: 


$$
\begin{aligned}
\mathrm{Q}= & \left(\Delta p_{1 \mu} \Delta p^{1 \mu}\right)^{1 / 2} \\
= & \frac{2 G m_{1} m_{2}}{b}\left[\mathrm{Q}^{1 \mathrm{PM}}+\frac{G}{b}\left(m_{1} \mathrm{Q}_{m_{1}}^{2 \mathrm{PM}}+m_{2} \mathrm{Q}_{m_{2}}^{2 \mathrm{PM}}\right)\right. \\
& \left.+\frac{G^{2}}{b^{2}}\left(m_{1}^{2} \mathrm{Q}_{m_{1}^{2}}^{3 \mathrm{PM}}+m_{1} m_{2} \mathrm{Q}_{m_{1} m_{2}}^{3 \mathrm{PM}}+m_{2}^{2} \mathrm{Q}_{m_{2}^{2}}^{3 \mathrm{PM}}\right)+\cdots\right]
\end{aligned}
$$

where each PM order is a homogeneous polynomial in the two masses. For nonspinning bodies, the Q's on the righthand side are functions only of energy (or velocity $v$ ). This mass dependence has been extended in Ref. [182] to include spin, such that

$$
\begin{aligned}
\mathrm{Q}_{m_{1}^{i} m_{2}^{j}}^{n \mathrm{PM}}= & \mathrm{Q}_{m_{1}^{i} m_{2}^{j}}^{n \mathrm{PM}}\left(v, \frac{a_{1}}{b}, \frac{a_{2}}{b}\right) \\
= & \mathrm{Q}_{m_{1}^{i} m_{2}^{j} a^{0}}^{n \mathrm{PM}}(v)+\frac{a_{1}}{b} \mathrm{Q}_{m_{1}^{i} m_{2}^{j} a_{1}}^{n \mathrm{PM}}(v) \\
& +\frac{a_{2}}{b} \mathrm{Q}_{m_{1}^{i} m_{2}^{j} a_{2}}^{n \mathrm{PM}}(v)+\mathcal{O}\left(a_{i}^{2}\right),
\end{aligned}
$$

where $b$ is the covariant impact parameter defined as the orthogonal distance between the incoming worldlines when using the covariant (Tulczyjew-Dixon) spinsupplementary condition $[195,196] \quad p_{\mathrm{i} \mu} S_{\mathrm{i}}^{\mu \nu}=0$. (See Refs. [85,86,182,197] for more details.)

The scattering angle $\chi$ by which the two bodies are deflected in the center-of-mass frame is related to $Q$ via [64]

$$
\sin \frac{\chi}{2}=\frac{\mathrm{Q}}{2 P_{\mathrm{c} . \mathrm{m} .}},
$$

where $P_{\text {c.m. }}$ is the magnitude of the total linear momentum in the center-of-mass frame and is given by

$$
P_{\text {c.m. }}=\frac{m_{1} m_{2}}{E} \sqrt{\gamma^{2}-1},
$$

where we recall that

$$
\begin{aligned}
E^{2} & =m_{1}^{2}+m_{2}^{2}+2 m_{1} m_{2} \gamma \\
& =M^{2}[1+2 \nu(\gamma-1)], \\
\gamma & =\frac{1}{\sqrt{1-v^{2}}} .
\end{aligned}
$$

Therefore, the scattering angle scaled by $E / m_{1} m_{2}$ has the same mass dependence as Q. (Equivalently, $\chi / \Gamma$ has the same mass dependence as $\mathrm{Q} / \mu$, where $\Gamma \equiv E / M$.)

For nonspinning binaries, and because of the symmetry under the exchange of the two bodies' labels, the mass dependence of $\chi / \Gamma$ can be written as a polynomial in the symmetric mass ratio $\nu$. This is because any homogeneous polynomial in the masses $\left(m_{1}, m_{2}\right)$ of degree $n$ can be written as polynomial in $\nu$ of degree $\lfloor n / 2\rfloor$. For example,

$$
\begin{aligned}
& c_{1} m_{1}^{3}+c_{2} m_{1}^{2} m_{2}+c_{2} m_{1} m_{2}^{2}+c_{1} m_{2}^{3} \\
& \quad=M^{3}\left[c_{1}+\left(c_{2}-3 c_{1}\right) \nu\right],
\end{aligned}
$$

for some mass-independent factors $c_{i}$. Hence, at each $n \mathrm{PM}$ order, $\chi / \Gamma$ is a polynomial in $\nu$ of degree $\lfloor(n-1) / 2\rfloor$.

When including spin, we also obtain a dependence on the antisymmetric mass ratio $\delta \equiv\left(m_{2}-m_{1}\right) / M$, since

$$
\begin{gathered}
a_{1}\left(c_{1} m_{1}^{3}+c_{2} m_{1}^{2} m_{2}+c_{3} m_{1} m_{2}^{2}+c_{4} m_{2}^{3}\right) \\
=M^{3} a_{1}\left(\alpha_{1}+\alpha_{2} \delta+\alpha_{3} \nu+\alpha_{4} \nu \delta\right),
\end{gathered}
$$

where $\alpha_{i}$ are some linear combinations of $c_{i}$.

Thus, we find that the scattering angle, up to 5PM order and to linear order in spin, has the following mass dependence:

$$
\chi=\chi_{a^{0}}+\chi_{a}+\mathcal{O}\left(a^{2}\right),
$$

$$
\begin{aligned}
\frac{\chi_{a^{0}}}{\Gamma}= & \frac{G M}{b} \mathrm{X}_{1}^{0}+\left(\frac{G M}{b}\right)^{2} \mathrm{X}_{2}^{0}+\left(\frac{G M}{b}\right)^{3}\left[\mathrm{X}_{3}^{0}+\nu \mathrm{X}_{3}^{0, \nu}\right] \\
& +\left(\frac{G M}{b}\right)^{4}\left[\mathrm{X}_{4}^{0}+\nu \mathrm{X}_{4}^{0, \nu}\right] \\
& +\left(\frac{G M}{b}\right)^{5}\left[\mathrm{X}_{5}^{0}+\nu \mathrm{X}_{5}^{0, \nu}+\nu^{2} \mathrm{X}_{5}^{0, \nu^{2}}\right]+\ldots,
\end{aligned}
$$

$$
\begin{aligned}
\frac{\chi_{a}}{\Gamma}= & \frac{a_{1}}{b}\left\{\frac{G M}{b} \mathrm{X}_{1}+\left(\frac{G M}{b}\right)^{2}\left[\mathrm{X}_{2}+\delta \mathrm{X}_{2}^{\delta}\right]\right. \\
& +\left(\frac{G M}{b}\right)^{3}\left[\mathrm{X}_{3}+\delta \mathrm{X}_{3}^{\delta}+\nu \mathrm{X}_{3}^{\nu}\right] \\
& +\left(\frac{G M}{b}\right)^{4}\left[\mathrm{X}_{4}+\delta \mathrm{X}_{4}^{\delta}+\nu \mathrm{X}_{4}^{\nu}+\nu \delta \mathrm{X}_{4}^{\nu \delta}\right] \\
& +\left(\frac{G M}{b}\right)^{5}\left[\mathrm{X}_{5}+\delta \mathrm{X}_{5}^{\delta}+\nu \mathrm{X}_{5}^{\nu}+\nu \delta \mathrm{X}_{5}^{\nu \delta}+\nu^{2} \mathrm{X}_{5}^{\nu^{2}}\right] \\
& +\ldots\}+1 \leftrightarrow 2,
\end{aligned}
$$

where the $X_{i} \cdots$ are functions only of energy/velocity. Since $\nu$ and $\nu \delta$ are of order $q$ when expanded in the mass ratio, their coefficients can be recovered from $1 \mathrm{SF}$ results.

This mass-ratio dependence holds for the total (local+ nonlocal) scattering angle. However, by choosing the split between the local and nonlocal parts as we did in Sec. II, i.e., by choosing the arbitrary length scale $s$ to be the radial distance $r$, we get the same mass-ratio dependence for the 
local part of the 5.5PN SO scattering angle. This is confirmed by the independent calculation of the nonlocal part of the scattering angle in Eq. (4.23) below, which is linear in $\nu$. (In Ref. [178], the authors introduced a "flexibility" factor in the relation between $s$ and $r$ to ensure that this mass-ratio dependence continues to hold at 5PN order for both the local and nonlocal contributions separately.)
Terms independent of $\nu$ in the scattering angle can be determined from the scattering angle of a spinning test particle in a Kerr background, which was calculated in Ref. [198]. For a test body with spin $s$ in a Kerr background with spin $a$, the 5PM test-body scattering angle to all PN orders and to linear order in spins can be obtained by integrating Eq. (65) of Ref. [198], leading to

$$
\begin{aligned}
\chi_{\text {test }}= & \frac{G M}{b}\left[\frac{2 v^{2}+2}{v^{2}}-\frac{4(a+s)}{b v}\right]+\pi\left(\frac{G M}{b}\right)^{2}\left[\frac{3\left(v^{2}+4\right)}{4 v^{2}}-\frac{\left(3 v^{2}+2\right)(4 a+3 s)}{2 b v^{3}}\right] \\
& +\left(\frac{G M}{b}\right)^{3}\left[\frac{2\left(5 v^{6}+45 v^{4}+15 v^{2}-1\right)}{3 v^{6}}-\frac{4\left(5 v^{4}+10 v^{2}+1\right)(3 a+2 s)}{b v^{5}}\right]+\pi\left(\frac{G M}{b}\right)^{4}\left[\frac{105\left(v^{4}+16 v^{2}+16\right)}{64 v^{4}}\right. \\
& \left.-\frac{21\left(5 v^{4}+20 v^{2}+8\right)(8 a+5 s)}{16 b v^{5}}\right]+\left(\frac{G M}{b}\right)^{5}\left[\frac{2\left(21 v^{10}+525 v^{8}+1050 v^{6}+210 v^{4}-15 v^{2}+1\right)}{5 v^{10}}\right. \\
& \left.-\frac{4\left(63 v^{8}+420 v^{6}+378 v^{4}+36 v^{2}-1\right)(5 a+3 s)}{3 b v^{9}}\right]+\mathcal{O}\left(G^{6}\right)+\mathcal{O}\left(a^{2}, a s, s^{2}\right) .
\end{aligned}
$$

Plugging this into Eq. (3.9) determines all of the $\mathrm{X}_{\mathbf{i}}(v)$ and $\mathrm{X}_{\mathrm{i}}^{\delta}(v)$ functions. Hence, we can write the 5PM SO part of the local scattering angle, expanded to 5.5PN order, as follows:

$$
\begin{aligned}
& \frac{\chi_{a}^{\mathrm{loc}}}{\Gamma}=\frac{a_{1}}{b}\left\{\left(\frac{G M}{v^{2} b}\right)(-4 v)+\pi\left(\frac{G M}{v^{2} b}\right)^{2}\left[\left(\frac{\delta}{2}-\frac{7}{2}\right) v+\left(\frac{3 \delta}{4}-\frac{21}{4}\right) v^{3}\right]\right. \\
& +\left(\frac{G M}{v^{2} b}\right)^{3}\left[\left(2 \delta-10+\mathrm{X}_{31}^{\nu} \nu\right) v+\left(20 \delta-100+\mathrm{X}_{33}^{\nu} \nu\right) v^{3}+\left(10 \delta-50+\mathrm{X}_{35}^{\nu} \nu\right) v^{5}+\mathrm{X}_{37}^{\nu} \nu v^{7}+\mathrm{X}_{39}^{\nu} \nu v^{9}\right] \\
& +\pi\left(\frac{G M}{v^{2} b}\right)^{4}\left[\left(\mathrm{X}_{41}^{\delta \nu} \delta \nu+\mathrm{X}_{41}^{\nu} \nu\right) v+\left(\frac{63}{4} \delta-\frac{273}{4}+\mathrm{X}_{43}^{\delta \nu} \delta \nu+\mathrm{X}_{43}^{\nu} \nu\right) v^{3}+\left(\frac{315}{8} \delta-\frac{1365}{8}+\mathrm{X}_{45}^{\delta \nu} \delta \nu+\mathrm{X}_{45}^{\nu} \nu\right) v^{5}\right. \\
& \left.+\left(\frac{315 \delta}{32}-\frac{1365}{32}+\mathbf{X}_{47}^{\delta \nu} \delta \nu+\mathbf{X}_{47}^{\nu} \nu\right) v^{7}+\left(\mathbf{X}_{49}^{\delta \nu} \delta \nu+\mathbf{X}_{49}^{\nu} \nu\right) v^{9}\right] \\
& +\left(\frac{G M}{v^{2} b}\right)^{5}\left[\left(-\frac{4 \delta}{3}+\frac{16}{3}+\mathrm{X}_{51}^{\delta \nu} \delta \nu+\mathrm{X}_{51}^{\nu} \nu+\mathrm{X}_{51}^{\nu^{2}} \nu^{2}\right) v+\left(48 \delta-192+\mathrm{X}_{53}^{\delta \nu} \delta \nu+\mathrm{X}_{53}^{\nu} \nu+\mathrm{X}_{53}^{\nu^{2}} \nu^{2}\right) v^{3}\right. \\
& +\left(504 \delta-2016+\mathrm{X}_{55}^{\delta \nu} \delta \nu+\mathrm{X}_{55}^{\nu} \nu+\mathrm{X}_{55}^{\nu^{2}} \nu^{2}\right) v^{5}+\left(560 \delta-2240+\mathrm{X}_{57}^{\delta \nu} \delta \nu+\mathrm{X}_{57}^{\nu} \nu+\mathrm{X}_{57}^{\nu^{2}} \nu^{2}\right) v^{7} \\
& \left.\left.+\left(84 \delta-336+\mathrm{X}_{59}^{\delta \nu} \delta \nu+\mathrm{X}_{59}^{\nu} \nu+\mathrm{X}_{59}^{\nu^{2}} \nu^{2}\right) v^{9}\right]\right\}+1 \leftrightarrow 2,
\end{aligned}
$$

where the $X_{i j}^{\nu}$ and $X_{i j}^{\delta \nu}$ coefficients are independent of the masses, and can be determined (as explained below) from $1 \mathrm{SF}$ results. The coefficient $X_{59}^{\nu^{2}}$ could be determined from future second-order self-force results.

\section{B. Relating the local Hamiltonian to the scattering angle}

The scattering angle can be calculated from the Hamiltonian by inverting the Hamiltonian and solving for $p_{r}(E, L, r)$, and then evaluating the integral

$$
\chi=-2 \int_{r_{0}}^{\infty} \frac{\partial p_{r}(E, L, r)}{\partial L} d r-\pi
$$

where $r_{0}$ is the turning point, obtained from the largest root of $p_{r}(E, L, r)=0 . E$ and $L$ represent the physical center-of-mass energy and canonical angular momentum, respectively.

As noted above, we express the scattering angle in terms of the covariant impact parameter $b$, but use the canonical angular momentum $L$ in the Hamiltonian (corresponding to 
the Newton-Wigner spin-supplementary condition). The two are related via $[85,86]$

$$
\begin{aligned}
L & =L_{\mathrm{cov}}+\Delta L, \\
L_{\mathrm{cov}} & =P_{\text {c.m. }} b=\frac{\mu}{\Gamma} \gamma v b, \\
\Delta L & =M \frac{\Gamma-1}{2}\left[a_{1}+a_{2}-\frac{\delta}{\Gamma}\left(a_{2}-a_{1}\right)\right],
\end{aligned}
$$

which can be used to replace $L$ with $b$ in the scattering angle. We can also replace $E$ with $v$ using Eq. (3.5).

Starting from the 4.5PN SO Hamiltonian, as given by Eq. (5.6) of Ref. [182], determines all of the unknown coefficients in the scattering angle in Eq. (3.12) up to that order. Writing an ansatz for the local 5.5PN part in terms of unknown coefficients, such as

$$
\begin{aligned}
& g_{S}^{5.5 \mathrm{PN}, \mathrm{loc}}=\frac{g_{04}}{r^{4}}+g_{23} \frac{p_{r}^{2}}{r^{3}}+g_{42} \frac{p_{r}^{4}}{r^{2}}+g_{61} \frac{p_{r}^{6}}{r}+g_{80} p_{r}^{8}, \\
& g_{S^{*}}^{5.5 \mathrm{PN}, \mathrm{loc}}=\frac{g_{04}^{*}}{r^{4}}+g_{23}^{*} \frac{p_{r}^{2}}{r^{3}}+g_{42}^{*} \frac{p_{r}^{4}}{r^{2}}+g_{61}^{*} \frac{p_{r}^{6}}{r}+g_{80}^{*} p_{r}^{8},
\end{aligned}
$$

calculating the scattering angle, and matching to Eq. (3.12) allows us to relate the ten unknowns in that ansatz to the six unknowns in the scattering angle at that order. This leads to

$$
\begin{aligned}
& g_{S}^{5.5 \mathrm{PN}, \mathrm{loc}}=2\left\{\frac { 1 } { r ^ { 4 } } \left[\nu\left(\frac{3 \mathrm{X}_{59}^{\delta \nu}}{32}-2 \mathrm{X}_{49}^{\delta \nu}-\frac{35 \mathrm{X}_{39}^{\nu}}{16}+2 \mathrm{X}_{49}^{\nu}-\frac{3 \mathrm{X}_{59}^{\nu}}{32}+\frac{309077}{1152}-\frac{35449 \pi^{2}}{3072}\right)+\nu^{2}\left(\frac{235111}{2304}-\frac{3 \mathrm{X}_{59}^{\nu^{2}}}{32}-\frac{583 \pi^{2}}{192}\right)\right.\right. \\
& \left.-\frac{\nu^{4}}{64}-\frac{413 \nu^{3}}{512}\right]+\frac{p_{r}^{2}}{r^{3}}\left[\frac{3 \nu^{4}}{8}-\frac{8259 \nu^{3}}{128}+\left(\frac{198133}{384}-\frac{1087 \pi^{2}}{128}\right) \nu^{2}+\nu\left(2 \mathbf{X}_{49}^{\delta \nu}+\frac{35 \mathbf{X}_{39}^{\nu}}{8}-2 \mathbf{X}_{49}^{\nu}+\frac{1125}{16}\right)\right] \\
& +\frac{p_{r}^{4}}{r^{2}}\left[-\frac{107 \nu^{4}}{64}-\frac{73547 \nu^{3}}{512}+\frac{31913 \nu^{2}}{256}+\nu\left(\frac{8597}{128}-\frac{35 \mathbf{X}_{39}^{\nu}}{24}\right)\right] \\
& \left.+\frac{p_{r}^{6}}{r}\left[\frac{1577 \nu^{4}}{320}-\frac{11397 \nu^{3}}{512}-\frac{2553 \nu^{2}}{256}-\frac{893 \nu}{256}\right]+p_{r}^{8}\left[\frac{189 \nu^{4}}{64}+\frac{945 \nu^{3}}{512}+\frac{99 \nu^{2}}{256}-\frac{27 \nu}{128}\right]\right\} \\
& g_{S^{*}}^{5.5 \mathrm{PN}, \mathrm{loc}}=\frac{3}{2}\left\{\frac { 1 } { r ^ { 4 } } \left[-\frac{15 \nu^{4}}{512}-\frac{111 \nu^{3}}{128}+\nu\left(2 \mathrm{X}_{49}^{\delta \nu}-\frac{3 \mathrm{X}_{59}^{\delta \nu}}{32}-\frac{35 \mathrm{X}_{39}^{\nu}}{16}+2 \mathrm{X}_{49}^{\nu}-\frac{3 \mathrm{X}_{59}^{\nu}}{32}+\frac{131519}{576}-\frac{90149 \pi^{2}}{12288}\right)-\frac{1701}{512}\right.\right. \\
& \left.+\nu^{2}\left(-\frac{3 X_{59}^{\nu^{2}}}{32}-\frac{123 \pi^{2}}{64}+\frac{29081}{512}\right)\right]+\frac{p_{r}^{2}}{r^{3}}\left[\frac{171 \nu^{4}}{256}-\frac{489 \nu^{3}}{8}+\left(\frac{77201}{256}-\frac{123 \pi^{2}}{16}\right) \nu^{2}-\frac{27}{64}\right. \\
& \left.+\nu\left(-2 X_{49}^{\delta \nu}+\frac{35 X_{39}^{\nu}}{8}-2 X_{49}^{\nu}+\frac{86897}{768}-\frac{27697 \pi^{2}}{2048}\right)\right]+\frac{p_{r}^{4}}{r^{2}}\left[-\frac{1377 \nu^{4}}{512}-\frac{13905 \nu^{3}}{128}+\frac{12135 \nu^{2}}{512}+\frac{2525}{512}\right. \\
& \left.+\nu\left(\frac{10569}{256}-\frac{35 \mathrm{X}_{39}^{\nu}}{24}\right)\right]+\frac{p_{r}^{6}}{r}\left[\frac{16077 \nu^{4}}{2560}-\frac{2391 \nu^{3}}{640}-\frac{879 \nu^{2}}{512}+\frac{77 \nu}{32}+\frac{3555}{512}\right] \\
& \left.+p_{r}^{8}\left[\frac{945 \nu^{4}}{512}+\frac{315 \nu^{3}}{128}+\frac{1053 \nu^{2}}{512}+\frac{189 \nu}{128}+\frac{693}{512}\right]\right\} \text {, }
\end{aligned}
$$

where we switched to dimensionless variables. We see that the five unknowns $\left(X_{39}^{\nu}, X_{49}^{\nu}, X_{49}^{\delta \nu}, X_{59}^{\nu}, X_{59}^{\delta \nu}\right)$ from the scattering angle only appear in the linear-in- $\nu$ coefficients of the gyro-gravitomagnetic factors up to order $p_{r}^{4}$, while the unknown $X_{59}^{\nu^{2}}$ only appears in the quadratic-in- $\nu$ coefficients of the circular-orbit $\left(1 / r^{4}\right)$ part. All other coefficients have been determined, due to the structure of the PMexpanded scattering angle, and from lower-order and testbody results.

\section{Redshift and precession frequency}

To determine the linear-in- $\nu$ coefficients in the local Hamiltonian from 1SF results, we calculate the redshift and spin-precession invariants from the total (local + nonlocal) Hamiltonian, since GSF calculations do not differentiate between the two, and then match their small-mass-ratio expansion to $1 \mathrm{SF}$ expressions known in the literature.

An important step in this calculation is the first law of binary mechanics, which was derived for nonspinning particles in circular orbits in Ref. [199], generalized to spinning particles in circular orbits in Ref. [200], to nonspinning particles in eccentric orbits in Refs. [201,202], and to spinning particles in eccentric orbits in Ref. [182]. It reads

$$
\mathrm{d} E=\Omega_{r} \mathrm{~d} I_{r}+\Omega_{\phi} \mathrm{d} L+\sum_{\mathrm{i}}\left(z_{\mathrm{i}} \mathrm{d} m_{\mathrm{i}}+\Omega_{S_{\mathrm{i}}} \mathrm{d} S_{\mathrm{i}}\right),
$$


where $\Omega_{r}$ and $\Omega_{\phi}$ are the radial and azimuthal frequencies, $I_{r}$ is the radial action, $z_{\mathrm{i}}$ is the redshift, and $\Omega_{S_{\mathrm{i}}}$ is the spinprecession frequency.

The orbit-averaged redshift is a gauge-invariant quantity that can be calculated from the Hamiltonian using

$$
z_{1}=\left\langle\frac{\partial H}{\partial m_{1}}\right\rangle=\frac{1}{T_{r}} \oint \frac{\partial H}{\partial m_{1}} d t
$$

where $T_{r}$ is the radial period. The spin-precession frequency $\Omega_{S_{1}}$ and spin-precession invariant $\psi_{1}$ are given by

$$
\begin{aligned}
\Omega_{S_{1}} & =\left\langle\frac{\partial H}{\partial S_{1}}\right\rangle=\frac{1}{T_{r}} \oint \frac{\partial H}{\partial S_{1}} d t, \\
\psi_{1} & \equiv \frac{\Omega_{S_{1}}}{\Omega_{\phi}} .
\end{aligned}
$$

In evaluating these integrals, we follow Refs. [134,141] in using the Keplerian parametrization for the radial variable

$$
r=\frac{1}{u_{p}(1+e \cos \xi)}
$$

where $u_{p}$ is the inverse of the semilatus rectum, $e$ is the eccentricity, and $\xi$ is the relativistic anomaly. The radial and azimuthal periods are calculated from the Hamiltonian using

$$
\begin{array}{r}
T_{r} \equiv \oint d t=2 \int_{0}^{\pi}\left(\frac{\partial H}{\partial p_{r}}\right)^{-1} \frac{d r}{d \xi} d \xi \\
T_{\phi} \equiv \oint d \phi=2 \int_{0}^{\pi} \frac{\partial H}{\partial L}\left(\frac{\partial H}{\partial p_{r}}\right)^{-1} \frac{d r}{d \xi} d \xi .
\end{array}
$$

Performing the above steps yields the redshift and spinprecession invariants in terms of the gauge-dependent $u_{p}$ and $e$, i.e., $z_{1}\left(u_{p}, e\right)$ and $\psi_{1}\left(u_{p}, e\right)$. We then express them in terms of the gauge-independent variables

$$
x \equiv\left(M \Omega_{\phi}\right)^{2 / 3}, \quad \imath \equiv \frac{3 x}{k},
$$

where $k \equiv T_{\phi} /(2 \pi)-1$ is the fractional periastron advance. The expressions we obtain for $z_{1}(x, l)$ and $\psi(x, l)$ agree up to $3.5 \mathrm{PN}$ order with those in Eq. (50) of Ref. [134] and Eq. (83) of Ref. [141], respectively.

Note that the denominator of $\imath$ in Eq. (3.23) is of order 1PN, which effectively scales down the PN ordering such that, to obtain the spin-precession invariant at fourthsubleading PN order, we need to include the 5PN nonspinning part of the Hamiltonian, which is given in Refs. [177,178].

\section{Comparison with self-force results}

Next, we expand the redshift $z_{1}(x, \imath)$ and spin-precession invariant $\psi_{1}(x, l)$ to first order in the mass ratio $q$, first order in the massive body's spin $a_{2} \equiv a$, and zeroth order in the spin of the smaller companion $a_{1}$. In doing so, we make use of another set of variables $(y, \lambda)$, defined by

$$
\begin{aligned}
& y \equiv\left(m_{2} \Omega_{\phi}\right)^{2 / 3}=\frac{x}{(1+q)^{2 / 3}}, \\
& \lambda \equiv \frac{3 y}{T_{\phi} /(2 \pi)-1}=\frac{l}{(1+q)^{2 / 3}},
\end{aligned}
$$

where the mass ratio $q=m_{1} / m_{2}$.

Schematically, those expansions have the following dependence on the scattering-angle unknowns:

$$
\begin{aligned}
z_{1}(y, \lambda) & =\cdots+q\left[\cdots+a\left\{\mathbf{X}_{39}^{\nu}, \mathbf{X}_{49}^{\nu}-\mathbf{X}_{49}^{\delta \nu}, \mathbf{X}_{59}^{\nu}-\mathbf{X}_{59}^{\delta \nu}\right\}\right] \\
\psi_{1}(y, \lambda) & =\cdots+q\left\{\mathbf{X}_{39}^{\nu}, \mathbf{X}_{49}^{\nu}+\mathbf{X}_{49}^{\delta \nu}, \mathbf{X}_{59}^{\nu}+\mathbf{X}_{59}^{\delta \nu}\right\}
\end{aligned}
$$

which can be seen from the structure of the scattering angle in Eq. (3.12). In those expressions, the $\mathcal{O}(a)$ part of the redshift depends on the unknown $X_{39}^{\nu}$ and the difference of the two pairs of unknowns $\left(\mathbf{X}_{49}^{\nu}, \mathbf{X}_{49}^{\delta \nu}\right)$ and $\left(\mathbf{X}_{59}^{\nu}, \mathbf{X}_{59}^{\delta \nu}\right)$, while the spin-precession invariant depends on $X_{39}^{\nu}$ and the sum of the two pairs of unknowns. This means that solving for $\mathrm{X}_{39}^{\nu}$ requires the $1 \mathrm{SF}$ result for either $z_{1}$ or $\psi_{1}$, while solving for the other unknowns requires both.

Hence, to solve for all five unknowns, we need at least three (or two) orders in eccentricity in the redshift, at first order in the Kerr spin, and two (or three) orders in eccentricity in the spin-precession invariant, at zeroth order in both spins. Equivalently, instead of the spinprecession invariant, one could use the redshift at linear order in the spin of the smaller body $a_{1}$, but that is known from 1SF results for circular orbits only [133]. Incidentally, the available $1 \mathrm{SF}$ results are just enough to solve for the five unknowns, since the redshift is known to $\mathcal{O}\left(e^{4}\right)$ $[132,134,203]$ and the spin-precession invariant to $\mathcal{O}\left(e^{2}\right)$ [139].

The last unknown $\mathrm{X}_{59}^{\nu^{2}}$ in the 5.5PN scattering angle appears in both the redshift and spin-precession invariants at second order in the mass ratio, thus requiring secondorder self-force results for circular orbits.

To compare $z_{1}(y, \lambda)$ and $\psi_{1}(y, \lambda)$ with GSF results, we write them in terms of the Kerr background values of the variables $(y, \lambda)$ expressed in terms of $\left(u_{p}, e\right)$. The relations between the two sets of variables are explained in detail in Appendix B of Ref. [182], and we just need to append to Eqs. (B16)-(B20) there the following PN terms: 


$$
\begin{aligned}
y\left(u_{p}, e\right) & =y_{0}\left(u_{p}, e\right)+a y_{a}\left(u_{p}, e\right)+\mathcal{O}\left(a^{2}\right) \\
\lambda\left(u_{p}, e\right) & =\lambda_{0}\left(u_{p}, e\right)+a \lambda_{a}\left(u_{p}, e\right)+\mathcal{O}\left(a^{2}\right) \\
y_{a}\left(u_{p}, e\right) & =\cdots+\left(\frac{4829 e^{4}}{12}-4984 e^{2}\right) u_{p}^{13 / 2}, \\
\lambda_{a}\left(u_{p}, e\right) & =\cdots+\left(\frac{4671}{8}+\frac{13959 e^{2}}{8}-\frac{19657 e^{4}}{12}\right) u_{p}^{11 / 2} .
\end{aligned}
$$

We obtain the following 1 SF part of the inverse redshift $U_{1} \equiv 1 / z_{1}$ and spin-precession invariant $\psi_{1}$ :

$$
\begin{aligned}
& U_{1}=U_{1 a^{0}}^{(0)}+a U_{1 a}^{(0)}+q\left(U_{1 a^{0}}^{1 \mathrm{SF}}+a U_{1 a}^{1 \mathrm{SF}}\right)+\mathcal{O}\left(q^{2}, a^{2}\right) \text {, } \\
& \psi_{1}=\psi_{1 a^{0}}^{(0)}+q \psi_{1 a^{0}}^{1 \mathrm{SF}}+\mathcal{O}\left(q^{2}, a\right), \\
& U_{1 a}^{1 \mathrm{SF}}=\left(3-\frac{7 e^{2}}{2}-\frac{e^{4}}{8}\right) u_{p}^{5 / 2}+\left(18-4 e^{2}-\frac{117 e^{4}}{4}\right) u_{p}^{7 / 2}+\left(87+\frac{287 e^{2}}{2}-\frac{6277 e^{4}}{16}\right) u_{p}^{9 / 2} \\
& +\left[\frac{3890}{9}-\frac{241 \pi^{2}}{96}+\left(\frac{5876}{3}-\frac{569 \pi^{2}}{64}\right) e^{2}+\left(\frac{2025 \pi^{2}}{128}-3547\right) e^{4}\right] u_{p}^{11 / 2} \\
& +\left[8 X_{49}^{\delta \nu}-\frac{3 X_{59}^{\delta \nu}}{8}+\frac{35 X_{39}^{\nu}}{4}-8 X_{49}^{\nu}+\frac{3 X_{59}^{\nu}}{8}+\frac{17917 \pi^{2}}{768}+\frac{2027413}{2880}+\frac{2336 \gamma_{E}}{15}+\frac{928 \ln 2}{3}+\frac{1168 \ln u_{p}}{15}\right. \\
& +e^{2}\left(\frac{4832 \ln u_{p}}{15}+24 \mathrm{X}_{49}^{\delta \nu}-\frac{21 \mathrm{X}_{59}^{\delta \nu}}{16}+\frac{175 \mathrm{X}_{39}^{\nu}}{8}-24 \mathrm{X}_{49}^{\nu}+\frac{21 \mathrm{X}_{59}^{\nu}}{16}+\frac{182411 \pi^{2}}{1536}+\frac{31389241}{2880}+\frac{9664 \gamma_{E}}{15}\right. \\
& -1728 \ln 2+2916 \ln 3)+e^{4}\left(-\frac{1248 \ln u_{p}}{5}-42 X_{49}^{\delta \nu}+\frac{63 X_{59}^{\delta \nu}}{32}-\frac{175 X_{39}^{\nu}}{4}+42 X_{49}^{\nu}-\frac{63 X_{59}^{\nu}}{32}-\frac{2496 \gamma_{E}}{5}\right. \\
& \left.\left.-\frac{200393 \pi^{2}}{1024}-\frac{137249131}{7680}+\frac{782912 \ln 2}{15}-\frac{328779 \ln 3}{10}\right)\right] u_{p}^{13 / 2} \\
& \psi_{1 a^{0}}^{1 \mathrm{SF}}=-u_{p}+\left(\frac{9}{4}+e^{2}\right) u_{p}^{2}+\left[\frac{739}{16}-\frac{123 \pi^{2}}{64}+\left(\frac{341}{16}-\frac{123 \pi^{2}}{256}\right) e^{2}\right] u_{p}^{3} \\
& +\left[\frac{628 \ln u_{p}}{15}+\frac{31697 \pi^{2}}{6144}-\frac{587831}{2880}+\frac{1256 \gamma_{E}}{15}+\frac{296}{15} \ln 2+\frac{729 \ln 3}{5}\right. \\
& \left.+e^{2}\left(\frac{268 \ln u_{p}}{5}-\frac{164123}{480}-\frac{23729 \pi^{2}}{4096}+\frac{536 \gamma_{E}}{5}+\frac{11720 \ln 2}{3}-\frac{10206 \ln 3}{5}\right)\right] u_{p}^{4} \\
& +\left[4 X_{49}^{\delta \nu}-\frac{3 X_{59}^{\delta \nu}}{16}-\frac{35 X_{39}^{\nu}}{8}+4 X_{49}^{\nu}-\frac{3 X_{59}^{\nu}}{16}+\frac{6793111 \pi^{2}}{24576}-\frac{22306 \gamma_{E}}{35}-\frac{115984853}{57600}+\frac{22058 \ln 2}{105}-\frac{31347 \ln 3}{28}\right. \\
& -\frac{11153}{35} \ln u_{p}+e^{2}\left(\frac{4248047}{4800}+18 \mathbf{X}_{49}^{\delta \nu}-\frac{15 \mathbf{X}_{59}^{\delta \nu}}{16}-\frac{35 \mathbf{X}_{39}^{\nu}}{2}+18 \mathbf{X}_{49}^{\nu}-\frac{15 \mathbf{X}_{59}^{\nu}}{16}+\frac{4895607 \pi^{2}}{16384}-\frac{22682 \gamma_{E}}{15}\right. \\
& \left.\left.+\frac{4430133 \ln 3}{320}+\frac{9765625 \ln 5}{1344}-\frac{4836254 \ln 2}{105}-\frac{11341 \ln u_{p}}{15}\right)\right] u_{p}^{5} \text {. }
\end{aligned}
$$

These results can be directly compared with those derived in GSF literature. In particular, for the redshift, we match to Eq. (4.1) of Ref. [132], Eq. (23) of Ref. [203], and Eq. (20) of Ref. [134], while for the precession frequency, we match to Eq. (3.33) of Ref. [139]. ${ }^{1}$ The matching to 1 SF results leads to the following solution for the unknown coefficients in the scattering angle:

\footnotetext{
${ }^{1}$ Note that the $\mathcal{O}\left(e^{2} u_{p}^{5}\right)$ term in Eq. (3.33) of Ref. [139] has a typo, but the correct expression is provided in the Black Hole Perturbation Toolkit [204].
} 


$$
\begin{aligned}
& X_{39}^{\nu}=\frac{26571}{1120}, \\
& X_{49}^{\delta \nu}=\frac{533669}{4800}-\frac{97585 \pi^{2}}{8192}, \\
& X_{49}^{\nu}=-\frac{403129}{4800}+\frac{80823 \pi^{2}}{8192}, \\
& X_{59}^{\delta \nu}=\frac{285673}{240}-\frac{2477 \pi^{2}}{16}, \\
& X_{59}^{\nu}=\frac{402799}{270}-\frac{4135 \pi^{2}}{144} .
\end{aligned}
$$

Note that all of the logarithms and Euler constants, which are purely nonlocal, cancel between GSF results and those in Eqs. (3.28) and (3.29), thus providing a good check for our calculations.

Another check would be possible once 1SF results are computed at higher orders in eccentricity, since one could directly compare them to our results for the redshift and spin-precession invariants that are provided in the Supplemental Material [183] expanded to $\mathcal{O}\left(e^{8}\right)$.

\section{E. Local scattering angle and Hamiltonian}

Inserting the solution from Eq. (3.30) into the scattering angle in Eq. (3.12) yields

$$
\begin{aligned}
\frac{\chi_{a}^{\mathrm{loc}}}{\Gamma}= & \frac{a_{1}}{b}\left\{\left(\frac{G M}{v^{2} b}\right)(-4 v)+\pi\left(\frac{G M}{v^{2} b}\right)^{2}\left[\left(\frac{\delta}{2}-\frac{7}{2}\right) v+\left(\frac{3 \delta}{4}-\frac{21}{4}\right) v^{3}\right]\right. \\
& +\left(\frac{G M}{v^{2} b}\right)^{3}\left[(2 \delta-10) v+(20 \delta-100+10 \nu) v^{3}+\left(10 \delta-50+\frac{77}{2} \nu\right) v^{5}+\frac{177}{4} \nu v^{7}+\frac{26571}{1120} \nu v^{9}\right] \\
& +\pi\left(\frac{G M}{v^{2} b}\right)^{4}\left[\left(\frac{63}{4} \delta-\frac{273}{4}-\frac{3}{4} \delta \nu+\frac{39}{4} \nu\right) v^{3}+\left(\frac{315}{8} \delta-\frac{1365}{8}-\frac{45}{8} \delta \nu+\frac{777}{8} \nu\right) v^{5}\right. \\
& +\left(\frac{315 \delta}{32}-\frac{1365}{32}+\left(-\frac{257}{96}-\frac{251 \pi^{2}}{256}\right) \delta \nu+\left(\frac{23717}{96}-\frac{733 \pi^{2}}{256}\right) \nu\right) v^{7} \\
& \left.+\left(\left(\frac{533669}{4800}-\frac{97585 \pi^{2}}{8192}\right) \delta \nu+\left(\frac{80823 \pi^{2}}{8192}-\frac{403129}{4800}\right) \nu\right) v^{9}\right] \\
& +\left(\frac{G M}{v^{2} b}\right)^{5}\left[\left(-\frac{4 \delta}{3}+\frac{16}{3}\right) v+(48 \delta-192-4 \delta \nu+32 \nu) v^{3}+\left(504 \delta-2016-109 \delta \nu+1032 \nu-16 \nu^{2}\right) v^{5}\right. \\
& +\left(560 \delta-2240+\left(-\frac{21995}{54}-\frac{80 \pi^{2}}{9}\right) \delta \nu+\left(\frac{150220}{27}-\frac{2755 \pi^{2}}{36}\right) \nu-168 \nu^{2}\right) v^{7} \\
& \left.\left.+\left(84 \delta-336+\left(\frac{285673}{240}-\frac{2477 \pi^{2}}{16}\right) \delta \nu+\left(\frac{402799}{270}-\frac{4135 \pi^{2}}{144}\right) \nu+\mathrm{X}_{59}^{\nu^{2}} \nu^{2}\right) v^{9}\right]\right\}+1 \leftrightarrow 2 .
\end{aligned}
$$

For the gyro-gravitomagnetic factors, which are one of the main results of this paper, substituting the solution (3.30) into Eq. (3.16) yields the following local part:

$$
\begin{aligned}
g_{S}^{5.5 \mathrm{PN}, \mathrm{loc}}= & 2\left\{\left[-\frac{\nu^{4}}{64}-\frac{413 \nu^{3}}{512}+\nu^{2}\left(-\frac{3 \mathrm{X}_{59}^{\nu^{2}}}{32}-\frac{583 \pi^{2}}{192}+\frac{235111}{2304}\right)+\left(\frac{62041 \pi^{2}}{3072}-\frac{11646877}{57600}\right) \nu\right] \frac{1}{r^{4}}\right. \\
& +\left[\frac{3 \nu^{4}}{8}-\frac{8259 \nu^{3}}{128}+\left(\frac{198133}{384}-\frac{1087 \pi^{2}}{128}\right) \nu^{2}+\left(\frac{3612403}{6400}-\frac{22301 \pi^{2}}{512}\right) \nu\right] \frac{p_{r}^{2}}{r^{3}} \\
& +\left[-\frac{107 \nu^{4}}{64}-\frac{73547 \nu^{3}}{512}+\frac{31913 \nu^{2}}{256}+\frac{8337 \nu}{256}\right] \frac{p_{r}^{4}}{r^{2}}+\left[\frac{1577 \nu^{4}}{320}-\frac{11397 \nu^{3}}{512}-\frac{2553 \nu^{2}}{256}-\frac{893 \nu}{256}\right] \frac{p_{r}^{6}}{r} \\
& \left.+\left[\frac{189 \nu^{4}}{64}+\frac{945 \nu^{3}}{512}+\frac{99 \nu^{2}}{256}-\frac{27 \nu}{128}\right] p_{r}^{8}\right\},
\end{aligned}
$$




$$
\begin{aligned}
g_{S^{*}}^{5.5 \mathrm{PN}, \mathrm{loc}}= & \frac{3}{2}\left\{\left[-\frac{5 \nu^{4}}{128}-\frac{37 \nu^{3}}{32}+\nu^{2}\left(-\frac{\mathrm{X}_{59}^{\nu^{2}}}{8}-\frac{41 \pi^{2}}{16}+\frac{29081}{384}\right)+\left(\frac{23663 \pi^{2}}{3072}-\frac{55}{2}\right) \nu-\frac{567}{128}\right] \frac{1}{r^{4}}\right. \\
& +\left[\frac{57 \nu^{4}}{64}-\frac{163 \nu^{3}}{2}+\left(\frac{77201}{192}-\frac{41 \pi^{2}}{4}\right) \nu^{2}+\left(\frac{34677}{160}-\frac{4829 \pi^{2}}{384}\right) \nu-\frac{9}{16}\right] \frac{p_{r}^{2}}{r^{3}} \\
& +\left[-\frac{459 \nu^{4}}{128}-\frac{4635 \nu^{3}}{32}+\frac{4045 \nu^{2}}{128}+\frac{107 \nu}{12}+\frac{2525}{384}\right] \frac{p_{r}^{4}}{r^{2}}+\left[\frac{5359 \nu^{4}}{640}-\frac{797 \nu^{3}}{160}-\frac{293 \nu^{2}}{128}+\frac{77 \nu}{24}+\frac{1185}{128}\right] \frac{p_{r}^{6}}{r} \\
& \left.+\left[\frac{315 \nu^{4}}{128}+\frac{105 \nu^{3}}{32}+\frac{351 \nu^{2}}{128}+\frac{63 \nu}{32}+\frac{231}{128}\right] p_{r}^{8}\right\} .
\end{aligned}
$$

\section{F. Comparison with numerical relativity}

To quantify the effect of the 5.5PN SO part on the dynamics, and that of the remaining unknown coefficient $\mathrm{X}_{59}^{\nu^{2}}$, we compare the binding energy calculated from the EOB Hamiltonian to NR. The binding energy provides a good diagnostic for the conservative dynamics of the binary system $[174,205,206]$, and can be calculated from accurate NR simulations by subtracting the radiated energy $E_{\text {rad }}$ from the Arnowitt-Deser-Misner (ADM) energy $E_{\mathrm{ADM}}$ at the beginning of the simulation [207], i.e.,

$$
\bar{E}_{\mathrm{NR}}=E_{\mathrm{ADM}}-E_{\mathrm{rad}}-M .
$$

To isolate the SO contribution $\bar{E}^{\mathrm{SO}}$ to the binding energy, we combine configurations with different spin orientations (parallel or antiparallel to the orbital angular momentum), as explained in Refs. [208,209]. One possibility is to use

$$
\bar{E}^{\mathrm{SO}}(\nu, \chi, \chi) \simeq \frac{1}{2}[\bar{E}(\nu, \chi, \chi)-\bar{E}(\nu,-\chi,-\chi)],
$$

where $\chi$ here is the magnitude of the dimensionless spin. This relation subtracts the nonspinning and spin-spin parts, with corrections remaining at order $\chi^{3}$, which provides a good approximation since the spin-cubed contribution to the binding energy is about an order of magnitude smaller than the SO contribution, as was shown in Ref. [209].

We calculate the binding energy for circular orbits from the EOB Hamiltonian using $\bar{E}_{\mathrm{EOB}}=H_{\mathrm{EOB}}-M$ while neglecting radiation reaction effects, which implies that $\bar{E}_{\mathrm{EOB}}$ is not expected to agree well with $\bar{E}_{\mathrm{NR}}$ near the end of the inspiral. We set $p_{r}=0$ in the Hamiltonian and numerically solve $\dot{p}_{r}=0=-\partial H / \partial r$ for the angular momentum $L$ at different orbital separations. Then, we plot $\bar{E}$ versus the dimensionless parameter

$$
v_{\Omega} \equiv(M \Omega)^{1 / 3},
$$

where the orbital frequency $\Omega=\partial H / \partial L$. Finally, we compare the EOB binding energy to NR data for the binding energy that were extracted in Ref. [209] from the Simulating eXtreme Spacetimes (SXS) catalog [210,211].
In particular, we use the simulations with SXS ID 0228 and 0215 for $q=1$, and 0291 and 0264 for $q=1 / 3$, all with spin magnitudes $\chi=0.6$ aligned and antialigned. The numerical error in these simulations is significantly smaller than the SO contribution to the binding energy.

In Fig. 1, we plot the relative difference in the SO contribution $\bar{E}^{\mathrm{SO}}$ between $\mathrm{EOB}$ and NR for two mass ratios, $q=1$ and $q=1 / 3$, as a function of $v_{\Omega}$ up to $v_{\Omega}=0.38$, which corresponds to about an orbit before merger. We see that the inclusion of the 5.5PN SO part (with the remaining unknown $X_{59}^{\nu^{2}}=0$ ) provides an improvement over $4.5 \mathrm{PN}$, but the difference is smaller than that between 3.5PN and 4.5PN. In addition, since the remaining unknown $X_{59}^{\nu^{2}}$ is expected to be about $\mathcal{O}\left(10^{2}\right)$, based on the other coefficients in the scattering angle, we plot the energy for $X_{59}^{\nu^{2}}=500$ and $X_{59}^{\nu^{2}}=-500$,

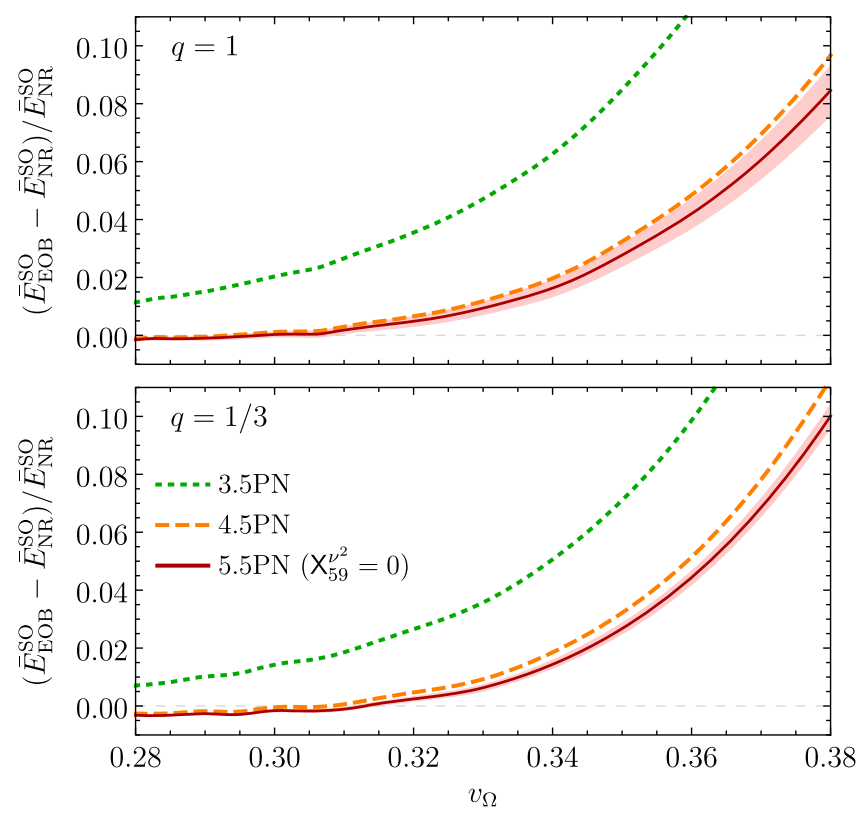

FIG. 1. Relative difference in the SO contribution to the binding energy between EOB and NR, plotted versus the frequency parameter $v_{\Omega}$. The 5.5PN curve corresponds to $\mathrm{X}_{59}^{\nu^{2}}=0$, while the upper and lower edges of the shaded region around it correspond to $X_{59}^{\nu^{2}}=-500$ and $X_{59}^{\nu^{2}}=500$, respectively. 
demonstrating that the effect of that unknown is less than the difference between 4.5PN and 5.5PN, with a decreasing effect for small mass ratios.

\section{NONLOCAL 5.5PN SO SCATTERING ANGLE}

The local part of the Hamiltonian and scattering angle calculated in the previous section is valid for both bound and unbound orbits. However, the nonlocal part of the Hamiltonian from Sec. II is only valid for bound orbits since it was calculated in a small-eccentricity expansion. In this section, we complement these results by calculating the nonlocal part for unbound orbits in a large-eccentricity (or large-angular-momentum) expansion.

The nonlocal part of the 4PN scattering angle was first computed in Ref. [212], in both the time and frequency domains, at leading order in the large-eccentricity expansion. This was extended in Ref. [178] at 5PN at leading order in eccentricity, and in Ref. [179] at 6PN to next-to-next-to-leading order in eccentricity. In addition, Refs. [213,214] recovered analytical expressions for the nonlocal scattering angle by using high-precision arithmetic methods.

It was shown in Ref. [212] that the nonlocal contribution to the scattering angle is given by

$$
\begin{gathered}
\chi_{\text {nonloc }}=\frac{1}{\nu} \frac{\partial}{\partial L} W_{\text {nonloc }}(E, L), \\
W_{\text {nonloc }}=\int d t \delta H_{\text {nonloc }},
\end{gathered}
$$

with $\delta H_{\text {nonloc }}$ given by Eq. (2.5), leading to

$$
\begin{gathered}
W_{\text {nonloc }}=W^{\text {flux split }}+W^{\text {flux }}, \\
W^{\text {flux split }}=-\frac{G M}{c^{3}} \int d t \operatorname{Pf}_{2 s / c} \int \frac{d \tau}{|\tau|} \mathcal{F}_{\mathrm{LO}}^{\text {split }}(t, t+\tau), \\
W^{\text {flux }}=\frac{2 G M}{c^{3}} \int d t \mathcal{F}_{\mathrm{LO}}(t, t) \ln \left(\frac{r}{s}\right) .
\end{gathered}
$$

To evaluate the integral in the large-eccentricity limit, we follow the steps used in Refs. [178,212]. We use the quasiKeplerian parametrization for hyperbolic motion [190,215]

$$
\begin{gathered}
r=\bar{a}_{r}\left(e_{r} \cosh \bar{u}-1\right), \\
\bar{n} t=e_{t} \sinh \bar{u}-\bar{u}, \\
\phi=2 K \arctan \left[\sqrt{\frac{e_{\phi}+1}{e_{\phi}-1}} \tanh \frac{\bar{u}}{2}\right],
\end{gathered}
$$

which is the analytic continuation of the parametrization for elliptic orbits in Eqs. (2.6)-(2.8). In Appendix. A 2, we summarize the relations for these quantities in terms of the energy and angular momentum.

We begin by expressing the variables $(r, \dot{\phi}, \dot{r})$, which enter the multipole moments, in terms of $\left(\phi, L, e_{t}\right)$, such that

$$
\begin{aligned}
r= & \frac{L^{2}}{1+e_{t} \cos \phi}+\frac{2 \delta \chi_{A}+(2-\nu) \chi_{S}}{L\left(e_{t} \cos \phi+1\right)^{2}} \\
& \times\left(2 \phi e_{t} \sin \phi+4 e_{t} \cos \phi+e_{t}^{2}+3\right), \\
\dot{\phi}= & \frac{\left(e_{t} \cos \phi+1\right)^{2}}{L^{3}}+\frac{\left(e_{t} \cos \phi+1\right)\left(2 \delta \chi_{A}+(2-\nu) \chi_{S}\right)}{2 L^{6}} \\
& \times\left(-8 \phi e_{t} \sin \phi+e_{t}^{2} \cos (2 \phi)-12 e_{t} \cos \phi-3 e_{t}^{2}-10\right), \\
\dot{r}= & \frac{e_{t} \sin \phi}{L}+\frac{e_{t}\left(2 \delta \chi_{A}+(2-\nu) \chi_{S}\right)}{2 L^{4}} \\
& \times\left(e_{t} \sin (2 \phi)-2 \sin \phi+4 \phi \cos \phi\right) .
\end{aligned}
$$

We then use these relations to obtain an exact expression for the flux-split function $\mathcal{F}_{\mathrm{LO}}^{\text {split }}\left(\phi, \phi^{\prime}\right)$, with no eccentricity expansion, which takes the form

$$
\begin{aligned}
\mathcal{F}_{\mathrm{LO}}^{\mathrm{split}}\left(\phi, \phi^{\prime}\right)= & \frac{4 \nu^{2}}{15 L^{10}}\left(1+e_{t} \cos \phi\right)^{2}\left(1+e_{t} \cos \phi^{\prime}\right)^{2} \\
& \times\left(F_{0}+F_{1} e_{t}+F_{2} e_{t}^{2}\right) \\
& +\frac{\nu^{2}}{L^{13}}\left(1+e_{t} \cos \phi\right)\left(1+e_{t} \cos \phi^{\prime}\right) \\
& \times\left(F_{0}^{s}+F_{1}^{s} e_{t}+\cdots+F_{6}^{s} e_{t}^{6}\right),
\end{aligned}
$$

where the functions $F_{\mathrm{i}}\left(\phi, \phi^{\prime}\right)$ are given by Eq. (92) of Ref. [83], but the functions $F_{\mathrm{i}}^{s}\left(\phi, \phi^{\prime}\right)$ in the SO part are too lengthy to write here. Instead, we expand $\mathcal{F}_{\mathrm{LO}}^{\text {split }}\left(\phi, \phi^{\prime}\right)$ to leading order in a large-eccentricity expansion (in powers of $\left.1 / e_{t}\right)$. To do that, we define the rescaled mean motion $\tilde{n} \equiv \bar{n} / e_{t}$, write Eq. (4.7) as

$$
\tilde{n} t=\sinh \bar{u}-\frac{\bar{u}}{e_{t}},
$$

and solve for $\bar{u}$ in a $1 / e_{t}$ expansion,

$$
\bar{u}=\sinh ^{-1}(t \tilde{n})+\frac{\sinh ^{-1}(t \tilde{n})}{e_{t} \sqrt{1+t^{2} \tilde{n}^{2}}}+\mathcal{O}\left(e_{t}^{-2}\right) .
$$

Substituting this into Eq. (4.8) and expanding yields

$$
\begin{aligned}
\phi(t)= & \tan ^{-1}(t \tilde{n})+\mathcal{O}\left(e_{t}^{-1}\right) \\
& -\frac{t \tilde{n} e_{t}\left[2 \delta \chi_{A}+(2-\nu) \chi_{S}\right]}{L^{3} \sqrt{t^{2} \tilde{n}^{2}+1}}+\mathcal{O}\left(e_{t}^{0}\right) .
\end{aligned}
$$

Defining $\tilde{t} \equiv \tilde{n} t$ and $\tilde{\tau} \equiv \tilde{n} \tau$, and then substituting into Eq. (4.10) and expanding yields 


$$
\begin{aligned}
\mathcal{F}^{\text {split }}(t, t+\tau)= & \frac{4 \nu^{2} e_{t}^{6}}{15 L^{10}} \frac{\mathrm{f}_{6}(\tilde{t}, \tilde{\tau})}{\left(\tilde{t}^{2}+1\right)^{5 / 2}\left(2 \tilde{\tau} \tilde{t}+\tilde{t}^{2}+\tilde{\tau}^{2}+1\right)^{5 / 2}}+\mathcal{O}\left(e_{t}^{5}\right) \\
& +\frac{4 \nu^{2} e_{t}^{8}}{15 L^{13}} \frac{\chi_{S} f_{8}^{S}(\tilde{t}, \tilde{\tau})+\delta \chi_{A} f_{8}^{A}(\tilde{t}, \tilde{\tau})}{\left(\tilde{t}^{2}+1\right)^{7 / 2}\left(2 \tilde{\tau} \tilde{t}+\tilde{t}^{2}+\tilde{\tau}^{2}+1\right)^{7 / 2}}+\mathcal{O}\left(e_{t}^{7}\right),
\end{aligned}
$$

with

$$
\begin{aligned}
\mathrm{f}_{6}(\tilde{t}, \tilde{\tau})= & 2 \tilde{t}^{6}+6 \tilde{\tau} \tilde{t}^{5}+\left(6 \tilde{\tau}^{2}+28\right) \tilde{t}^{4}+2 \tilde{\tau}\left(\tilde{\tau}^{2}+28\right) \tilde{t}^{3}+\left(39 \tilde{\tau}^{2}+50\right) \tilde{t}^{2}+\tilde{\tau}\left(11 \tilde{\tau}^{2}+50\right) \tilde{t}-12\left(\tilde{\tau}^{2}-2\right), \\
\mathrm{f}_{8}^{S}(\tilde{t}, \tilde{\tau})= & 4(9 \nu-5) \tilde{t}^{8}+16(9 \nu-5) \tilde{\tau} \tilde{t}^{7}+2 \tilde{t}^{6}\left[18 \nu\left(6 \tilde{\tau}^{2}+5\right)-63 \tilde{\tau}^{2}-67\right]+2 \tilde{\tau} \tilde{t}^{5}\left[18 \nu\left(4 \tilde{\tau}^{2}+15\right)-49 \tilde{\tau}^{2}-201\right] \\
& +\tilde{t}^{4}\left[18 \nu\left(2 \tilde{\tau}^{4}+35 \tilde{\tau}^{2}+18\right)-2\left(19 \tilde{\tau}^{4}+206 \tilde{\tau}^{2}+141\right)\right]-2 \tilde{\tau} \tilde{t}^{3}\left[-36 \nu\left(5 \tilde{\tau}^{2}+9\right)+3 \tilde{\tau}^{4}+77 \tilde{\tau}^{2}+282\right] \\
& +\tilde{t}^{2}\left[3 \nu\left(35 \tilde{\tau}^{4}+130 \tilde{\tau}^{2}+84\right)-2\left(8 \tilde{\tau}^{4}+169 \tilde{\tau}^{2}+121\right)\right]+\tilde{\tau} \tilde{t}\left[3 \nu\left(5 \tilde{\tau}^{4}+22 \tilde{\tau}^{2}+84\right)-2\left(3 \tilde{\tau}^{4}+28 \tilde{\tau}^{2}+121\right)\right] \\
& +22 \tilde{\tau}^{4}-52 \tilde{\tau}^{2}-74-12 \nu\left(3 \tilde{\tau}^{4}+2 \tilde{\tau}^{2}-6\right), \\
\mathrm{f}_{8}^{A}(\tilde{t}, \tilde{\tau})= & -2\left(\tilde{t}^{2}+1\right)\left[40 \tilde{\tau} \tilde{t}^{5}+\left(63 \tilde{\tau}^{2}+57\right) \tilde{t}^{4}+7 \tilde{\tau}\left(7 \tilde{\tau}^{2}+23\right) \tilde{t}^{3}+\left(19 \tilde{\tau}^{4}+143 \tilde{\tau}^{2}+84\right) \tilde{t}^{2}+\tilde{\tau}\left(3 \tilde{\tau}^{4}+28 \tilde{\tau}^{2}+121\right) \tilde{t}\right. \\
& \left.+10 \tilde{t}^{6}-11 \tilde{\tau}^{4}+26 \tilde{\tau}^{2}+37\right] .
\end{aligned}
$$

Then, we evaluate the partie finie integral in Eq. (4.4), after writing it in terms of $\tilde{T} \equiv 2 \tilde{n} s / c$, to obtain

$$
\begin{aligned}
- & \operatorname{Pf}_{\tilde{T}} \int \frac{d \tilde{\tau}}{|\tilde{\tau}|} \mathcal{F}_{\mathrm{LO}}^{\text {split }}(t, t+\tau) \\
= & \frac{8 \nu^{2} e_{t}^{6}}{15 L^{10}\left(\tilde{t}^{2}+1\right)^{3}}\left[36+7 \tilde{t}^{2}+2\left(\tilde{t}^{2}+12\right) \ln \left(\frac{\tilde{T}}{2 \tilde{t}^{2}+2}\right)\right] \\
& +\frac{16 \nu^{2} e_{t}^{8}}{15 L^{13}\left(\tilde{t}^{2}+1\right)^{4}}\left\{\chi_{S}\left[(3 \nu-5) \tilde{t}^{4}+(36 \nu+5) \tilde{t}^{2}-\left(2(5-9 \nu) \tilde{t}^{2}-36 \nu+37\right) \ln \left(\frac{\tilde{T}}{2 \tilde{t}^{2}+2}\right)+60 \nu-53\right]\right. \\
& \left.-\delta \chi_{A}\left[\left(10 \tilde{t}^{2}+37\right) \ln \left(\frac{\tilde{T}}{2 \tilde{t}^{2}+2}\right)+5 \tilde{t}^{4}-5 \tilde{t}^{2}+53\right]\right\} .
\end{aligned}
$$

Integrating over $t$, we obtain the flux-split potential

$$
\begin{aligned}
W^{\text {flux split }}= & \frac{2 \pi \nu^{2}}{15 e_{t}^{3} a_{r}^{7 / 2}}\left[100+37 \ln \left(\frac{s}{4 e_{t} a_{r}^{3 / 2}}\right)\right]+\frac{\pi \nu^{2}}{30 e_{t}^{4} a_{r}^{5}}\left\{31 \delta \chi_{A}\left[-137-46 \ln \left(\frac{s}{4 a_{r}^{3 / 2} e_{t}}\right)\right]\right. \\
& \left.+\chi_{S}\left[2774 \nu-4247-2(713-457 \nu) \ln \left(\frac{s}{4 a_{r}^{3 / 2} e_{t}}\right)\right]\right\} .
\end{aligned}
$$

The second contribution $W^{\text {flux }}$ in Eq. (4.5) can be easily integrated to yield

$$
\begin{aligned}
W^{\text {flux }}= & \frac{2 \pi \nu^{2}}{15 e_{t}^{3} a_{r}^{7 / 2}}\left[-\frac{85}{4}-37 \ln \left(\frac{s}{2 a_{r} e_{t}}\right)\right]+\frac{\pi \nu^{2}}{60 e_{t}^{4} a_{r}^{5}}\left\{\delta \chi_{A}\left[2255+2852 \ln \left(\frac{s}{2 a_{r} e_{t}}\right)\right]\right. \\
& \left.+\chi_{S}\left[2255-1365 \nu+(2852-1828 \nu) \ln \left(\frac{s}{2 a_{r} e_{t}}\right)\right]\right\},
\end{aligned}
$$

where we used

$$
r(t)=\frac{L^{2} \sqrt{\tilde{t}^{2}+1}}{e_{t}}+\frac{1}{L}\left[2 \delta \chi_{A}+(2-\nu) \chi_{S}\right]
$$


Adding the two contributions leads to

$$
\begin{aligned}
W^{\text {nonloc }}= & -\frac{\pi \nu^{2}}{30 e_{t}^{3} a_{r}^{7 / 2}}\left[74 \ln \left(4 a_{r}\right)-315\right] \\
& +\frac{\pi \nu^{2}}{60 e_{t}^{4} a_{r}^{5}}\left\{\chi_{S}[4183 \nu-6239\right. \\
& \left.+2(713-457 \nu) \ln \left(4 a_{r}\right)\right] \\
& \left.+\delta \chi_{A}\left[-6239+1426 \ln \left(4 a_{r}\right)\right]\right\},
\end{aligned}
$$

where we see that $s$ cancels. In terms of the energy and angular momentum, and expanding in $1 / L$ to leading order,

$$
\begin{aligned}
W^{\text {nonloc }}= & \frac{2 \pi \nu^{2} \bar{E}^{2}}{15 L^{3}}\left[315+74 \ln \left(\frac{\bar{E}}{2}\right)\right] \\
& +\frac{2 \pi \nu^{2} \bar{E}^{3}}{15 L^{4}}\left\{(4183 \nu-6239) \chi_{S}-6239 \delta \chi_{A}\right. \\
& \left.-2\left[713 \delta \chi_{A}+(713-457 \nu) \chi_{S}\right] \ln \left(\frac{E}{2}\right)\right\},
\end{aligned}
$$

and the nonlocal part of the scattering angle

$$
\begin{aligned}
\chi^{\text {nonloc }}= & -\frac{2 \pi \nu \bar{E}^{2}}{5 L^{4}}\left[315+74 \ln \left(\frac{\bar{E}}{2}\right)\right] \\
& -\frac{8 \pi \nu \bar{E}^{3}}{15 L^{5}}\left\{(4183 \nu-6239) \chi_{S}-6239 \delta \chi_{A}\right. \\
& \left.-2\left[713 \delta \chi_{A}+(713-457 \nu) \chi_{S}\right] \ln \left(\frac{E}{2}\right)\right\} .
\end{aligned}
$$

In terms of $b$ and $v$, using Eqs. (3.14) and (3.5),

$$
\begin{aligned}
\chi^{\text {nonloc }}= & -\frac{\pi \nu}{10 b^{4}}\left[148 \ln \left(\frac{v}{2}\right)+315\right] \\
& -\frac{\pi \nu v}{15 b^{5}}\left\{4 \ln \left(\frac{v}{2}\right)\left[(679 \nu-824) \chi_{S}-824 \delta \chi_{A}\right]\right. \\
& \left.+(6073 \nu-7184) \chi_{S}-7184 \delta \chi_{A}\right\} .
\end{aligned}
$$

\section{GAUGE-INVARIANT QUANTITIES FOR BOUND ORBITS}

In this section, we obtain two gauge-invariant quantities that characterize bound orbits: the radial action as a function of the energy and angular momentum, and the binding energy for circular orbits as a function of the orbital frequency.

\section{A. Radial action}

The radial action function contains the same gaugeinvariant information as the Hamiltonian, and from it several other functions can be derived that describe bound orbits, such as the periastron advance, which can be directly related to the scattering angle via analytic continuation $[77,88]$. This means that the entire calculation in Sec. III could be performed using the radial action instead of the Hamiltonian, as was done in Ref. [182].

The radial action is defined by the integral

$$
I_{r}=\frac{1}{2 \pi} \oint p_{r} d r
$$

and we split it into a local contribution and a nonlocal one, such that

$$
I_{r}=I_{r}^{\mathrm{loc}}+I_{r}^{\mathrm{nonloc}} .
$$

We calculate the local part from the local EOB Hamiltonian, i.e., Eq. (2.20) with the nonlocal parts of the potentials and gyro-gravitomagnetic factors set to zero. We invert the local Hamiltonian iteratively to obtain $p_{r}(\varepsilon, L, r)$ in a PN expansion, where we recall that

$$
\begin{aligned}
H_{\mathrm{EOB}} & =\frac{1}{\nu} \sqrt{1+2 \nu(\gamma-1)}, \\
\varepsilon & \equiv \gamma^{2}-1,
\end{aligned}
$$

with $\varepsilon<0, \gamma<1$ for bound orbits. Then, we integrate

$$
I_{r}=\frac{1}{\pi} \int_{r_{-}}^{r_{+}} p_{r}(\varepsilon, L, r) d r
$$

where $r_{ \pm}$are the zeros of the Newtonian-order $p_{r}^{(0)}=\sqrt{\varepsilon+2 / r-L^{2} / r^{2}}$, which are given by

$$
r_{ \pm}=\frac{1 \pm \sqrt{1+L^{2} \varepsilon}}{-\varepsilon} .
$$

It is convenient to express the radial action in terms of the covariant angular momentum $L_{\mathrm{cov}}=L-\Delta L$, with $\Delta L$ given by Eq. (3.14), since it can then be directly related to the coefficients of the scattering angle, as discussed in Ref. [182], and leads to slightly simpler coefficients for the SO part.

We obtain for the local part

$$
\begin{aligned}
I_{r}^{\mathrm{loc}}= & -L+I_{0}+\frac{I_{1}}{\Gamma L_{\mathrm{cov}}}+\frac{I_{2}^{s}}{\left(\Gamma L_{\mathrm{cov}}\right)^{2}} \\
& +\frac{I_{3}}{\left(\Gamma L_{\mathrm{cov}}\right)^{3}}+\frac{I_{4}^{s}}{\left(\Gamma L_{\mathrm{cov}}\right)^{4}}+\frac{I_{5}}{\left(\Gamma L_{\mathrm{cov}}\right)^{5}}+\frac{I_{6}^{s}}{\left(\Gamma L_{\mathrm{cov}}\right)^{6}} \\
& +\frac{I_{7}}{\left(\Gamma L_{\mathrm{cov}}\right)^{7}}+\frac{I_{8}^{s}}{\left(\Gamma L_{\mathrm{cov}}\right)^{8}}+\frac{I_{9}}{\left(\Gamma L_{\mathrm{cov}}\right)^{9}}+\frac{I_{10}^{s}}{\left(\Gamma L_{\mathrm{cov}}\right)^{10}},
\end{aligned}
$$

where each term starts at a given $\mathrm{PN}$ order, with $0.5 \mathrm{PN}$ order corresponding to each power in $1 / L$. Also, as noted 
in Ref. [178], when the radial action is written in this form, in terms of $\Gamma$, the coefficients $I_{2 n+1}^{(s)}$ become simple polynomials in $\nu$ of degree $\lfloor n\rfloor$.

The coefficients $I_{n}$ for the nonspinning local radial action up to 5PN order are given by Eq. (13.20) of Ref. [178]. The SO coefficients $I_{n}^{s}$ were derived in Ref. [182] to the 4.5PN order, but we list them here for completeness. The coefficients $I_{0}, I_{1}, I_{2}^{s}$ are exact, and are given by

$$
\begin{aligned}
I_{0} & =\frac{1+2 \varepsilon}{\sqrt{-\varepsilon}}, \\
I_{1} & =\frac{3}{4}(4+5 \varepsilon), \\
I_{2}^{s} & =-\frac{1}{4} \gamma(5 \varepsilon+2)\left(4 a_{b}+3 a_{t}\right),
\end{aligned}
$$

where $a_{b} \equiv S / M, a_{t} \equiv S^{*} / M$. The other SO coefficients, up to $5.5 \mathrm{PN}$, read

$$
\begin{aligned}
& I_{4}^{s}=-\frac{21}{64} \gamma\left(33 \varepsilon^{2}+36 \varepsilon+8\right)\left(8 a_{b}+5 a_{t}\right)+\gamma \nu\left\{\frac{21 a_{b}}{8}+\frac{9 a_{t}}{4}+\varepsilon\left(\frac{495 a_{b}}{16}+\frac{219 a_{t}}{8}\right)+\varepsilon^{2}\left[\left(\frac{17423}{192}-\frac{241 \pi^{2}}{512}\right) a_{b}\right.\right. \\
& \left.\left.+\left(\frac{2759}{32}-\frac{123 \pi^{2}}{128}\right) a_{t}\right]-\varepsilon^{3}\left[\left(\frac{156133}{3200}-\frac{22301 \pi^{2}}{4096}\right) a_{b}+\left(\frac{8381 \pi^{2}}{16384}-\frac{6527}{960}\right) a_{t}\right]+\mathcal{O}\left(\varepsilon^{4}\right)\right\}, \\
& I_{6}^{s}=\left(-\frac{25 \nu^{2}}{8}+\frac{1755 \nu}{16}-\frac{495}{2}\right) a_{b}+\left(-\frac{45 \nu^{2}}{16}+\frac{165 \nu}{2}-\frac{1155}{8}\right) a_{t} \\
& -\varepsilon\left\{\left[\frac{645 \nu^{2}}{8}+\left(\frac{3665 \pi^{2}}{256}-\frac{39715}{24}\right) \nu+\frac{3465}{2}\right] a_{b}+\left[\frac{1185 \nu^{2}}{16}+\left(\frac{1845 \pi^{2}}{128}-\frac{10305}{8}\right) \nu+\frac{8085}{8}\right] a_{t}\right\} \\
& +\varepsilon^{2}\left\{a_{b}\left(\left(\frac{10640477}{1920}-\frac{176785 \pi^{2}}{2048}\right) \nu+\nu^{2}\left(\frac{45 X_{59}^{\nu^{2}}}{128}+\frac{2755 \pi^{2}}{512}-\frac{176815}{384}\right)-\frac{121275}{32}\right)\right. \\
& \left.+a_{t}\left(\left(\frac{433715}{96}-\frac{1748755 \pi^{2}}{16384}\right) \nu+\nu^{2}\left(\frac{45 X_{59}^{\nu^{2}}}{128}+\frac{5535 \pi^{2}}{1024}-\frac{26175}{64}\right)-\frac{282975}{128}\right)\right\}+\mathcal{O}\left(\varepsilon^{3}\right), \\
& I_{8}^{s}=\left[\frac{455 \nu^{3}}{128}-\frac{10185 \nu^{2}}{32}+\left(\frac{3755465}{1152}-\frac{42875 \pi^{2}}{1536}\right) \nu-\frac{25025}{8}\right] a_{b} \\
& +\left[\frac{105 \nu^{3}}{32}-\frac{16485 \nu^{2}}{64}+\left(\frac{437605}{192}-\frac{1435 \pi^{2}}{64}\right) \nu-\frac{225225}{128}\right] a_{t} \\
& +\varepsilon\left\{a_{b}\left[\frac{4935 \nu^{3}}{32}+\left(\frac{8263591}{180}-\frac{9948785 \pi^{2}}{12288}\right) \nu+\nu^{2}\left(\frac{105 X_{59}^{\nu^{2}}}{64}-\frac{1583995}{192}+\frac{27895 \pi^{2}}{256}\right)-\frac{225225}{8}\right]\right. \\
& \left.+a_{t}\left[\frac{1155 \nu^{3}}{8}+\left(\frac{4594121}{144}-\frac{29957165 \pi^{2}}{49152}\right) \nu+\nu^{2}\left(\frac{105 X_{59}^{\nu^{2}}}{64}-\frac{209195}{32}+\frac{47355 \pi^{2}}{512}\right)-\frac{2027025}{128}\right]\right\}+\mathcal{O}\left(\varepsilon^{2}\right), \\
& I_{10}^{s}=a_{b}\left[-\frac{63 \nu^{4}}{16}+\frac{90405 \nu^{3}}{128}+\left(\frac{88995311}{1280}-\frac{545853 \pi^{2}}{512}\right) \nu+\nu^{2}\left(\frac{189 X_{59}^{\nu^{2}}}{128}+\frac{109515 \pi^{2}}{512}-\frac{1119461}{64}\right)-\frac{1322685}{32}\right] \\
& +a_{t}\left[-\frac{945 \nu^{4}}{256}+\frac{38115 \nu^{3}}{64}+\left(\frac{14456349}{320}-\frac{11632089 \pi^{2}}{16384}\right) \nu+\nu^{2}\left(\frac{189 X_{59}^{\nu^{2}}}{128}+\frac{167895 \pi^{2}}{1024}-\frac{3292149}{256}\right)-\frac{2909907}{128}\right] \\
& +\mathcal{O}(\varepsilon) \text {. }
\end{aligned}
$$

The nonlocal part can be calculated similarly by starting from the total Hamiltonian, expanding Eq. (5.1) in eccentricity, and then subtracting the local part. Alternatively, it can be calculated directly from the nonlocal Hamiltonian via [179]

$$
I_{r}^{\text {nonloc }}=-\frac{H_{\text {nonloc }}}{\Omega_{r}},
$$

where $\Omega_{r}=2 \pi / T_{r}$ is the radial frequency given by Eq. (A8).
The nonlocal Hamiltonian $H_{\text {nonloc }}$ in Eq. (2.19) is expressed in terms of $\left(e_{t}, a_{r}\right)$, but we can use Eqs. (A6) and (A12) to obtain $I_{r}^{\text {nonloc }}(E, L)$, i.e., as a function of energy and angular momentum. Then, we replace $E$ with $\left(e_{t}, L\right)$ using Eq. (A10), expand in eccentricity to $\mathcal{O}\left(e_{t}^{8}\right)$, and revert back to $(E, L)$. This way, we obtain an expression for $I_{r}^{\text {nonloc }}$ in powers of $1 / L$ that is valid to eighth order in eccentricity, and in which each $\varepsilon^{n}$ contributes up to order $e^{2 n}$. 
The result for the 4PN and 5.5PN SO contributions reads

$$
\begin{aligned}
& \frac{I_{r}^{\text {nonloc }}}{\nu}=\frac{1}{L_{\mathrm{cov}}^{7}}\left(\frac{170 \ln L_{\mathrm{cov}}}{3}-\frac{170 \gamma_{E}}{3}+\frac{18299}{96}-\frac{4777903}{90} \ln 2+\frac{13671875 \ln 5}{512}-\frac{15081309 \ln 3}{2560}\right) \\
& +\frac{\varepsilon}{L_{\mathrm{cov}}^{5}}\left(\frac{244 \ln L_{\mathrm{cov}}}{5}-\frac{244 \gamma_{E}}{5}+\frac{157823}{360}-\frac{10040414}{45} \ln 2+\frac{126953125 \ln 5}{1152}-\frac{13542147 \ln 3}{640}\right) \\
& +\frac{\varepsilon^{2}}{L_{\mathrm{cov}}^{3}}\left(\frac{74 \ln L_{\mathrm{cov}}}{15}-\frac{74 \gamma_{E}}{15}+\frac{89881}{240}-\frac{5292281}{15} \ln 2+\frac{130859375 \ln 5}{768}-\frac{35029179 \ln 3}{1280}\right) \\
& +\frac{\varepsilon^{3}}{L_{\mathrm{cov}}}\left(\frac{6187}{40}-\frac{11186786}{45} \ln 2+\frac{44921875 \ln 5}{384}-\frac{1878147 \ln 3}{128}\right) \\
& +\varepsilon^{4} L_{\mathrm{cov}}\left(\frac{40253}{1440}-\frac{1185023}{18} \ln 2+\frac{138671875 \ln 5}{4608}-\frac{6591861 \ln 3}{2560}\right) \\
& +a_{b}\left[\frac{1}{L_{\mathrm{cov}}^{10}}\left(-\frac{12579 \ln L_{\mathrm{cov}}}{5}-\frac{24068101}{2880}+\frac{12579 \gamma_{E}}{5}+\frac{398742736 \ln 2}{135}+\frac{281496303 \ln 3}{1024}-\frac{40131484375 \ln 5}{27648}\right)\right. \\
& +\frac{\varepsilon}{L_{\mathrm{cov}}^{8}}\left(-2499 \ln L_{\mathrm{cov}}-\frac{13345921}{720}+2499 \gamma_{E}+\frac{1548980449 \ln 2}{135}+\frac{1132984827 \ln 3}{1280}-\frac{38230234375 \ln 5}{6912}\right) \\
& +\frac{\varepsilon^{2}}{L_{\mathrm{cov}}^{6}}\left(-537 \ln L_{\mathrm{cov}}-\frac{7268749}{480}+537 \gamma_{E}+\frac{149780983 \ln 2}{9}+\frac{2572548417 \ln 3}{2560}-\frac{36141484375 \ln 5}{4608}\right) \\
& +\frac{\varepsilon^{3}}{L_{\mathrm{cov}}^{4}}\left(-13 \ln L_{\mathrm{cov}}-\frac{4143337}{720}+13 \gamma_{E}+\frac{1439288647 \ln 2}{135}+\frac{116812287 \ln 3}{256}-\frac{33865234375 \ln 5}{6912}\right) \\
& \left.+\frac{\varepsilon^{4}}{L_{\mathrm{cov}}^{2}}\left(-\frac{2608213}{2880}+\frac{342877711 \ln 2}{135}+\frac{318592683 \ln 3}{5120}-\frac{31401484375 \ln 5}{27648}\right)\right] \\
& +a_{t}\left[\frac{1}{L_{\mathrm{cov}}^{10}}\left(-\frac{8673 \ln L_{\mathrm{cov}}}{5}-\frac{16708517}{2880}+\frac{8673 \gamma_{E}}{5}+\frac{582216271 \ln 2}{270}+\frac{980901819 \ln 3}{5120}-\frac{29135234375 \ln 5}{27648}\right)\right. \\
& +\frac{\varepsilon}{L_{\mathrm{cov}}^{8}}\left(-\frac{5593 \ln L_{\mathrm{cov}}}{3}-\frac{1921829}{144}+\frac{5593 \gamma_{E}}{3}+\frac{231474971 \ln 2}{27}+\frac{806618331 \ln 3}{1280}-\frac{28420234375 \ln 5}{6912}\right) \\
& +\frac{\varepsilon^{2}}{L_{\mathrm{cov}}^{6}}\left(-455 \ln L_{\mathrm{cov}}-\frac{5444717}{480}+455 \gamma_{E}+\frac{191532524 \ln 2}{15}+\frac{1877210721 \ln 3}{2560}-\frac{9203828125 \ln 5}{1536}\right) \\
& +\frac{\varepsilon^{3}}{L_{\mathrm{cov}}^{4}}\left(-\frac{69 \ln L_{\mathrm{cov}}}{5}-\frac{3226241}{720}+\frac{69 \gamma_{E}}{5}+\frac{227762869 \ln 2}{27}+\frac{87726159 \ln 3}{256}-\frac{26708984375 \ln 5}{6912}\right) \\
& \left.+\frac{\varepsilon^{4}}{L_{\mathrm{cov}}^{2}}\left(-\frac{2112181}{2880}+\frac{562665401 \ln 2}{270}+\frac{49433247 \ln 3}{1024}-\frac{25712734375 \ln 5}{27648}\right)\right] \text {. }
\end{aligned}
$$

\section{B. Circular-orbit binding energy}

Here, we calculate the gauge-invariant binding energy $\bar{E}$ analytically in a PN expansion, as opposed to the numerical calculation in Sec. III F for the EOB binding energy.

For circular orbits and aligned spins, $\bar{E}$ can be calculated from the Hamiltonian (2.20) by setting $p_{r}=0$ and perturbatively solving $\dot{p}_{r}=0=-\partial H / \partial r$ for the angular momentum $L(r)$. Then, solving $\Omega=\partial H / \partial L$ for $r(\Omega)$, and substituting into the Hamiltonian yields $\bar{E}$ as a function of the orbital frequency. It is convenient to express $\bar{E}$ in terms of the dimensionless frequency parameter $v_{\Omega} \equiv\left(M \Omega_{\phi}\right)^{1 / 3}$. 
The nonspinning 4PN binding energy is given by Eq. (5.5) of Ref. [9], and the 4.5PN SO part is given by Eq. (5.11) of Ref. [182]. We obtain for the 5.5PN SO part

$$
\begin{aligned}
\bar{E}^{5.5 \mathrm{PN}, \mathrm{SO}}= & \nu v_{\Omega}^{13}\left\{S \left[-\frac{4725}{32}+\nu\left(\frac{1411663}{640}-\frac{10325 \pi^{2}}{64}+\frac{352 \gamma_{E}}{3}+\frac{2080 \ln 2}{9}\right)+\frac{352}{3} \nu \ln v_{\Omega}+\frac{310795 \nu^{3}}{5184}+\frac{35 \nu^{4}}{1458}\right.\right. \\
& \left.+\nu^{2}\left(\frac{5 \mathrm{X}_{59}^{\nu^{2}}}{8}+\frac{2425 \pi^{2}}{864}-\frac{1975415}{5184}\right)\right]+S^{*}\left[-\frac{2835}{128}+\nu\left(\frac{126715}{144}-\frac{102355 \pi^{2}}{1536}+\frac{160 \gamma_{E}}{3}+\frac{992 \ln 2}{9}\right)\right. \\
& \left.\left.+\frac{160}{3} \nu \ln v_{\Omega}+\nu^{2}\left(\frac{5 \mathrm{X}_{59}^{\nu^{2}}}{8}-\frac{205 \pi^{2}}{576}-\frac{275245}{3456}\right)+\frac{46765 \nu^{3}}{864}+\frac{875 \nu^{4}}{31104}\right]\right\} .
\end{aligned}
$$

\section{CONCLUSIONS}

Improving the spin description in waveform models is crucial for GW observations with the continually increasing sensitivities of the Advanced LIGO, Virgo, and KAGRA detectors [216], and for future GW detectors, such as the Laser Interferometer Space Antenna [217], the Einstein Telescope [218], the DECi-hertz Interferometer Gravitational wave Observatory [219], and Cosmic Explorer [220]. More accurate waveform models can lead to better estimates for the spins of binary systems, and for the orthogonal component of spin in precessing systems, which helps in identifying their formation channels $[4,5]$. For this purpose, in this paper we extended the SO coupling to the 5.5PN level.

We employed an approach [177,182] that combines several analytical approximation methods to obtain arbitrary-mass-ratio PN results from first-order self-force results. We computed the nonlocal-in-time contribution to the dynamics for bound orbits in a small-eccentricity expansion [Eq. (2.24)] and for unbound motion in a large-eccentricity expansion [Eq. (4.23)]. To our knowledge, this is the first time that nonlocal contributions to the conservative dynamics have been computed in the spin sector. For the local-in-time contribution, we exploited the simple mass-ratio dependence of the PM-expanded scattering angle and related the Hamiltonian coefficients to those of the scattering angle. This allowed us to determine all of the unknowns at that order from first-order self-force results, except for one unknown at second order in the mass ratio; see Eqs. (3.31)-(3.33). We also provided the radial action (in Sec. VA) and the circular-orbit binding energy [in Eq. (5.11)] as two important gauge-invariant quantities for bound orbits. We stress again that, although all calculations in this paper were performed for aligned spins, the SO coupling is applicable for generic precessing spins.

The local part of the 5.5PN SO coupling still has an unknown coefficient, but as we showed in Fig. 1, its effect on the dynamics is smaller than the difference between the 4.5 and 5.5PN orders. Determining that unknown could be done through targeted PN calculations, as was illustrated in
Ref. [98], in which the authors related the two missing coefficients at 5PN order to coefficients that can be calculated from an effective field theory approach. Alternatively, one could use analytical second-order selfforce results, which might become available in the near future, given the recent work on numerically computing the binding energy and energy flux $[142,143]$. Until then, one could still use the partial 5.5PN SO results in EOB waveform models complemented by NR calibration. Such an implementation would be straightforward, since we obtained the gyro-gravitomagnetic factors that enter directly into the SEOBNR [162-164] and TEOBResumS $[150,166,169]$ waveform models, and less directly into the IMRPhenom models [221-224], which are used in GW analyses.

\section{ACKNOWLEDGMENTS}

I am grateful to Alessandra Buonanno, Jan Steinhoff, and Justin Vines for fruitful discussions and for their invaluable feedback on earlier drafts of this paper. I also thank Sergei Ossokine for providing NR data for the binding energy, and thank the anonymous referee for useful suggestions.

\section{APPENDIX: QUASI-KEPLERIAN PARAMETRIZATION}

\section{Elliptic orbits}

For a binary in a bound orbit in the orbital plane, and using polar coordinates $(r, \phi)$, the quasi-Keplerian parametrization [190], up to $1.5 \mathrm{PN}$, reads

$$
\begin{gathered}
r=a_{r}\left(1-e_{r} \cos u\right), \\
\ell \equiv n t=u-e_{t} \sin u, \\
\phi=2 K \arctan \left[\sqrt{\frac{1+e_{\phi}}{1-e_{\phi}}} \tan \frac{u}{2}\right],
\end{gathered}
$$

where $a_{r}$ is the semimajor axis, $u$ is the eccentric anomaly, $\ell$ is the mean anomaly, $n$ is the mean motion (radial angular 
frequency), $K$ is the periastron advance, and $\left(e_{r}, e_{t}, e_{\phi}\right)$ are the radial, time, and phase eccentricities. Spin was included in the quasi-Keplerian parametrization in Refs. [192,193]. (See Fig. 2 of Ref. [193] for a geometric picture for some of these quantities.)

The (dimensionless) harmonic-coordinates Hamiltonian with LO SO reads

$$
H=\frac{c^{2}}{\nu}+\frac{p^{2}}{2}-\frac{1}{r}+\frac{L}{c^{3} r^{3}}\left[2 \delta \chi_{A}-(\nu-2) \chi_{S}\right] .
$$

By inserting $r=a_{r}\left(1-e_{r} \cos u\right)$ into the Hamiltonian at periastron $(u=0)$ and apastron $(u=\pi)$, one can solve for the energy and angular momentum (with $p_{r}=0$ ) as a function of $a_{r}$ and $e_{r}$, i.e.,

$$
\begin{aligned}
\bar{E} & =\frac{-1}{2 a_{r}}+\frac{(\nu-2) \chi_{S}-2 \delta \chi_{A}}{2 \sqrt{a_{r}^{5}\left(1-e_{r}^{2}\right)}}, \\
L & =\sqrt{a_{r}\left(1-e_{r}^{2}\right)}+\frac{\left(e_{r}^{2}+3\right)\left[2 \delta \chi_{A}+(2-\nu) \chi_{S}\right]}{2 a_{r}\left(e_{r}^{2}-1\right)},
\end{aligned}
$$

where $\bar{E} \equiv E-1 / \nu<0$ is the dimensionless binding energy, which is negative for bound orbits, and we only include the LO nonspinning and SO terms. These expansions can be inverted to obtain $e_{r}(\bar{E}, L)$ and $a_{r}(\bar{E}, L)$, leading to

$$
\begin{aligned}
& e_{r}=\sqrt{1+2 \bar{E} L^{2}}+\frac{4 E\left(1+\bar{E} L^{2}\right)\left[2 \delta \chi_{A}+(2-\nu) \chi_{S}\right]}{L \sqrt{1+2 \bar{E} L^{2}}}, \\
& a_{r}=\frac{-1}{2 \bar{E}}+\frac{2 \delta \chi_{A}+(2-\nu) \chi_{S}}{L} .
\end{aligned}
$$

The radial period $T_{r}$ and periastron advance $K$ can be calculated from the integrals

$$
\begin{aligned}
T_{r} & =\oint \frac{d r}{\dot{r}}=2 \int_{r_{p}}^{r_{a}} \frac{d r}{\partial H / \partial p_{r}}, \\
K & =\frac{1}{2 \pi} \oint d r \frac{\dot{\phi}}{\dot{r}}=2 \int_{r_{p}}^{r_{a}} d r \frac{\partial H / \partial L}{\partial H / \partial p_{r}},
\end{aligned}
$$

where $r_{p}$ and $r_{a}$ are the periastron and apastron separations calculated from the solution of $p_{r}=0$, which yields the PN expansion

$$
\begin{aligned}
n & =\frac{2 \pi}{T_{r}}=2 \sqrt{2}(-\bar{E})^{3 / 2} \\
& =\frac{1}{a_{r}^{3 / 2}}+\frac{3\left[2 \delta \chi_{A}+(2-\nu) \chi_{S}\right]}{2 a_{r}^{3} \sqrt{1-e_{t}^{2}}}, \\
K & =1+\frac{2(\nu-2) \chi_{S}-4 \delta \chi_{A}}{L^{3}} \\
& =1-\frac{4 \delta \chi_{A}+2(2-\nu) \chi_{S}}{a_{r}^{3 / 2}\left(1-e_{t}^{2}\right)^{3 / 2}} .
\end{aligned}
$$

The three eccentricities $\left(e_{r}, e_{t}, e_{\phi}\right)$ agree at $\mathrm{LO}$, and can be related to each other, and to the energy and angular momentum, via

$$
\begin{gathered}
e_{t}=\sqrt{1+2 \bar{E} L^{2}}+\frac{2 \bar{E}\left[2 \delta \chi_{A}+(2-\nu) \chi_{S}\right]}{L \sqrt{1+2 \bar{E} L^{2}}}, \\
e_{\phi}=\sqrt{1+2 \bar{E} L^{2}}+\frac{4 \bar{E}\left(1+\bar{E} L^{2}\right)\left[2 \delta \chi_{A}+(2-\nu) \chi_{S}\right]}{L \sqrt{1+2 \bar{E} L^{2}}}, \\
\frac{e_{r}}{e_{t}}=1+\frac{2 \bar{E}}{L}\left[2 \delta \chi_{A}+(2-\nu) \chi_{S}\right] \\
\frac{e_{\phi}}{e_{t}}=1+\frac{2 \bar{E}}{L}\left[2 \delta \chi_{A}+(2-\nu) \chi_{S}\right] .
\end{gathered}
$$

\section{Hyperbolic motion}

The quasi-Keplerian parametrization for hyperbolic motion [190,215] can be summarized, up to $1.5 \mathrm{PN}$, by the following equations:

$$
\begin{gathered}
r=\bar{a}_{r}\left(e_{r} \cosh \bar{u}-1\right), \\
\bar{n} t=e_{t} \sinh \bar{u}-\bar{u}, \\
\phi=2 K \arctan \left[\sqrt{\frac{e_{\phi}+1}{e_{\phi}-1}} \tanh \frac{\bar{u}}{2}\right] .
\end{gathered}
$$

The equations for hyperbolic motion are related to the elliptic-orbit equations via analytic continuation from $\bar{E}<0$ to $\bar{E}>0$, and $u \rightarrow i \bar{u}$ [190]. In particular, the energy and angular momentum are given by

$\bar{E}=\frac{1}{2 \bar{a}_{r}}+\frac{(\nu-2) \chi_{S}-2 \delta \chi_{A}}{2 \sqrt{\bar{a}_{r}^{5}\left(e_{r}^{2}-1\right)}}$,

$L=\sqrt{\bar{a}_{r}\left(e_{r}^{2}-1\right)}-\frac{\left(e_{r}^{2}+3\right)\left[2 \delta \chi_{A}+(2-\nu) \chi_{S}\right]}{2 \bar{a}_{r}\left(e_{r}^{2}-1\right)}$.

Inverting these expansions, we obtain

$$
\bar{a}_{r}=\frac{1}{2 \bar{E}}-\frac{2 \delta \chi_{A}+(2-\nu) \chi_{S}}{L},
$$

and $e_{r}(\bar{E}, L)$ is the same as in Eq. (A6).

The mean motion $\bar{n}$ and periastron advance $K$ are given by

$$
\begin{aligned}
\bar{n} & =\frac{2 \pi}{T_{r}}=2 \sqrt{2} \bar{E}^{3 / 2} \\
& =\frac{1}{\bar{a}_{r}^{3 / 2}}-\frac{3\left[2 \delta \chi_{A}+(2-\nu) \chi_{S}\right]}{2 \bar{a}_{r}^{3} \sqrt{e_{t}^{2}-1}},
\end{aligned}
$$




$$
\begin{aligned}
K & =1+\frac{2(\nu-2) \chi_{S}-4 \delta \chi_{A}}{L^{3}} \\
& =1-\frac{4 \delta \chi_{A}+2(2-\nu) \chi_{S}}{\bar{a}_{r}^{3 / 2}\left(e_{t}^{2}-1\right)^{3 / 2}}
\end{aligned}
$$

The eccentricities $e_{t}$ and $e_{\phi}$ are given in terms of energy and angular momentum by Eqs. (A10) and (A11), respectively.

[1] B. P. Abbott et al. (LIGO Scientific and Virgo Collaborations), Observation of Gravitational Waves from a Binary Black Hole Merger, Phys. Rev. Lett. 116, 061102 (2016).

[2] R. Abbott et al. (LIGO Scientific and Virgo Collaborations), GWTC-2: Compact Binary Coalescences Observed by LIGO and Virgo During the First Half of the Third Observing Run, Phys. Rev. X 11, 021053 (2021).

[3] B. P. Abbott et al. (LIGO Scientific and Virgo Collaborations), GWTC-1: A Gravitational-Wave Transient Catalog of Compact Binary Mergers Observed by LIGO and Virgo during the First and Second Observing Runs, Phys. Rev. X 9, 031040 (2019).

[4] B. P. Abbott et al. (LIGO Scientific and Virgo Collaborations), Binary black hole population properties inferred from the first and second observing runs of Advanced LIGO and Advanced Virgo, Astrophys. J. Lett. 882, L24 (2019).

[5] R. Abbott et al. (LIGO Scientific and Virgo Collaborations), Population properties of compact objects from the second LIGO-Virgo gravitational-wave transient catalog, Astrophys. J. Lett. 913, L7 (2021).

[6] L. Blanchet and G. Faye, General relativistic dynamics of compact binaries at the third post-Newtonian order, Phys. Rev. D 63, 062005 (2001).

[7] P. Jaranowski and G. Schaefer, Third post-Newtonian higher order ADM Hamilton dynamics for two-body point mass systems, Phys. Rev. D 57, 7274 (1998); 63, 029902 (E) (2000).

[8] M.E. Pati and C. M. Will, Post-Newtonian gravitational radiation and equations of motion via direct integration of the relaxed Einstein equations. 1. Foundations, Phys. Rev. D 62, 124015 (2000).

[9] T. Damour, P. Jaranowski, and G. Schäfer, Nonlocal-intime action for the fourth post-Newtonian conservative dynamics of two-body systems, Phys. Rev. D 89, 064058 (2014).

[10] T. Damour, P. Jaranowski, and G. Schäfer, Fourth postNewtonian effective one-body dynamics, Phys. Rev. D 91, 084024 (2015).

[11] T. Damour, P. Jaranowski, and G. Schäfer, Conservative dynamics of two-body systems at the fourth post-Newtonian approximation of general relativity, Phys. Rev. D 93, 084014 (2016).

[12] L. Bernard, L. Blanchet, G. Faye, and T. Marchand, Center-of-mass equations of motion and conserved integrals of compact binary systems at the fourth postNewtonian order, Phys. Rev. D 97, 044037 (2018).
[13] T. Marchand, L. Bernard, L. Blanchet, and G. Faye, Ambiguity-free completion of the equations of motion of compact binary systems at the fourth post-Newtonian order, Phys. Rev. D 97, 044023 (2018).

[14] S. Foffa and R. Sturani, Conservative dynamics of binary systems to fourth post-Newtonian order in the EFT approach I: Regularized Lagrangian, Phys. Rev. D 100, 024047 (2019).

[15] S. Foffa, R. A. Porto, I. Rothstein, and R. Sturani, Conservative dynamics of binary systems to fourth postNewtonian order in the EFT approach II: Renormalized Lagrangian, Phys. Rev. D 100, 024048 (2019).

[16] J. Blümlein, A. Maier, P. Marquard, and G. Schäfer, Fourth post-Newtonian Hamiltonian dynamics of two-body systems from an effective field theory approach, Nucl. Phys. B955, 115041 (2020).

[17] J. Blümlein, A. Maier, P. Marquard, and G. Schäfer, The fifth-order post-Newtonian Hamiltonian dynamics of twobody systems from an effective field theory approach: Potential contributions, Nucl. Phys. B965, 115352 (2021).

[18] F. Larrouturou, Q. Henry, L. Blanchet, and G. Faye, The quadrupole moment of compact binaries to the fourth postNewtonian order: I. Non-locality in time and infra-red divergencies, arXiv:2110.02240.

[19] J. Blümlein, A. Maier, P. Marquard, and G. Schäfer, The fifth-order post-Newtonian Hamiltonian dynamics of twobody systems from an effective field theory approach, arXiv:2110.13822.

[20] G. L. Almeida, S. Foffa, and R. Sturani, Tail contributions to gravitational conservative dynamics, arXiv:2110.14146.

[21] W. Tulczyjew, Equations of motion of rotating bodies in general relativity theory, Acta Phys. Polon 18, 37 (1959).

[22] H. Tagoshi, A. Ohashi, and B. J. Owen, Gravitational field and equations of motion of spinning compact binaries to 2.5 post-Newtonian order, Phys. Rev. D 63, 044006 (2001).

[23] R. A. Porto, Post-Newtonian corrections to the motion of spinning bodies in NRGR, Phys. Rev. D 73, 104031 (2006).

[24] G. Faye, L. Blanchet, and A. Buonanno, Higher-order spin effects in the dynamics of compact binaries. I. Equations of motion, Phys. Rev. D 74, 104033 (2006).

[25] L. Blanchet, A. Buonanno, and G. Faye, Higher-order spin effects in the dynamics of compact binaries. II. Radiation field, Phys. Rev. D 74, 104034 (2006); 75, 049903(E) (2007); 81, 089901(E) (2010). 
[26] T. Damour, P. Jaranowski, and G. Schaefer, Hamiltonian of two spinning compact bodies with next-to-leading order gravitational spin-orbit coupling, Phys. Rev. D 77, 064032 (2008).

[27] L. Blanchet, A. Buonanno, and G. Faye, Tail-induced spinorbit effect in the gravitational radiation of compact binaries, Phys. Rev. D 84, 064041 (2011).

[28] J. Hartung and J. Steinhoff, Next-to-next-to-leading order post-Newtonian spin-orbit Hamiltonian for self-gravitating binaries, Ann. Phys. (N.Y.) 523, 783 (2011).

[29] D. L. Perrodin, Subleading spin-orbit correction to the Newtonian potential in effective field theory formalism, in Proceedings of the 12th Marcel Grossmann Meeting on General Relativity (2010), pp. 725-727, arXiv:1005.0634.

[30] R. A. Porto, Next to leading order spin-orbit effects in the motion of inspiralling compact binaries, Classical Quantum Gravity 27, 205001 (2010).

[31] J. Hartung, J. Steinhoff, and G. Schafer, Next-to-next-toleading order post-Newtonian linear-in-spin binary Hamiltonians, Ann. Phys. (N.Y.) 525, 359 (2013).

[32] S. Marsat, A. Bohe, G. Faye, and L. Blanchet, Next-tonext-to-leading order spin-orbit effects in the equations of motion of compact binary systems, Classical Quantum Gravity 30, 055007 (2013).

[33] A. Bohe, S. Marsat, G. Faye, and L. Blanchet, Next-tonext-to-leading order spin-orbit effects in the near-zone metric and precession equations of compact binaries, Classical Quantum Gravity 30, 075017 (2013).

[34] M. Levi and J. Steinhoff, Next-to-next-to-leading order gravitational spin-orbit coupling via the effective field theory for spinning objects in the post-Newtonian scheme, J. Cosmol. Astropart. Phys. 01 (2016) 011.

[35] M. Levi, A. J. Mcleod, and M. Von Hippel, $\mathrm{N}^{3} \mathrm{LO}$ gravitational spin-orbit coupling at order $G^{4}$, J. High Energy Phys. 07 (2021) 115.

[36] S. Hergt and G. Schäfer, Higher-order-in-spin interaction Hamiltonians for binary black holes from Poincare invariance, Phys. Rev. D 78, 124004 (2008).

[37] R. A. Porto and I. Z. Rothstein, Calculation of the First Nonlinear Contribution to the General-Relativistic SpinSpin Interaction for Binary Systems, Phys. Rev. Lett. 97, 021101 (2006).

[38] R. A. Porto and I. Z. Rothstein, Next to leading order spin (1)spin(1) effects in the motion of inspiralling compact binaries, Phys. Rev. D 78, 044013 (2008); 81, 029905(E) (2010).

[39] R. A. Porto and I. Z. Rothstein, Spin(1)spin(2) effects in the motion of inspiralling compact binaries at third order in the post-Newtonian expansion, Phys. Rev. D 78, 044012 (2008); 81, 029904(E) (2010).

[40] S. Hergt, J. Steinhoff, and G. Schäfer, Reduced Hamiltonian for next-to-leading order spin-squared dynamics of general compact binaries, Classical Quantum Gravity 27, 135007 (2010).

[41] J. Hartung and J. Steinhoff, Next-to-next-to-leading order post-Newtonian $\operatorname{spin}(1)-\operatorname{spin}(2)$ Hamiltonian for self-gravitating binaries, Ann. Phys. (N.Y.) 523, 919 (2011).

[42] M. Levi, Binary dynamics from spin1-spin2 coupling at fourth post-Newtonian order, Phys. Rev. D 85, 064043 (2012).
[43] M. Levi and J. Steinhoff, Next-to-next-to-leading order gravitational spin-squared potential via the effective field theory for spinning objects in the post-Newtonian scheme, J. Cosmol. Astropart. Phys. 01 (2016) 008.

[44] G. Cho, B. Pardo, and R. A. Porto, Gravitational radiation from inspiralling compact objects: Spin-spin effects completed at the next-to-leading post-Newtonian order, Phys. Rev. D 104, 024037 (2021).

[45] M. Levi and J. Steinhoff, Leading order finite size effects with spins for inspiralling compact binaries, J. High Energy Phys. 06 (2015) 059.

[46] M. Levi and J. Steinhoff, Complete conservative dynamics for inspiralling compact binaries with spins at the fourth post-Newtonian order, J. Cosmol. Astropart. Phys. 09 (2021) 029.

[47] M. Levi, S. Mougiakakos, and M. Vieira, Gravitational cubic-in-spin interaction at the next-to-leading postNewtonian order, J. High Energy Phys. 01 (2021) 036.

[48] M. Levi and F. Teng, NLO gravitational quartic-in-spin interaction, J. High Energy Phys. 01 (2021) 066.

[49] J. Vines and J. Steinhoff, Spin-multipole effects in binary black holes and the test-body limit, Phys. Rev. D 97, 064010 (2018).

[50] N. Siemonsen and J. Vines, Test black holes, scattering amplitudes and perturbations of Kerr spacetime, Phys. Rev. D 101, 064066 (2020).

[51] T. Futamase and Y. Itoh, The post-Newtonian approximation for relativistic compact binaries, Living Rev. Relativity 10, 2 (2007).

[52] L. Blanchet, Gravitational radiation from post-Newtonian sources and inspiralling compact binaries, Living Rev. Relativity 17, 2 (2014).

[53] G. Schäfer and P. Jaranowski, Hamiltonian formulation of general relativity and post-Newtonian dynamics of compact binaries, Living Rev. Relativity 21, 7 (2018).

[54] M. Levi and J. Steinhoff, Spinning gravitating objects in the effective field theory in the post-Newtonian scheme, J. High Energy Phys. 09 (2015) 219.

[55] R. A. Porto, The effective field theorist's approach to gravitational dynamics, Phys. Rep. 633, 1 (2016).

[56] M. Levi, Effective field theories of post-Newtonian gravity: A comprehensive review, Rep. Prog. Phys. 83, 075901 (2020).

[57] K. Westpfahl and M. Goller, Gravitational scattering of two relativistic particles in postlinear approximation, Lett. Nuovo Cimento Soc. Ital. Fis. 26, 573 (1979).

[58] K. Westpfahl and H. Hoyler, Gravitational bremsstrahlung in post-linear fast-motion approximation, Lett. Nuovo Cimento Soc. Ital. Fis. 27, 581 (1980).

[59] L. Bel, T. Damour, N. Deruelle, J. Ibanez, and J. Martin, Poincaré-invariant gravitational field and equations of motion of two pointlike objects: The postlinear approximation of general relativity, Gen. Relativ. Gravit. 13, 963 (1981).

[60] G. Schäfer, The ADM Hamiltonian at the postlinear approximation, Gen. Relativ. Gravit. 18, 255 (1986).

[61] T. Ledvinka, G. Schaefer, and J. Bicak, Relativistic Closed-Form Hamiltonian for Many-Body Gravitating Systems in the Post-Minkowskian Approximation, Phys. Rev. Lett. 100, 251101 (2008). 
[62] T. Damour, Gravitational scattering, post-Minkowskian approximation and effective one-body theory, Phys. Rev. D 94, 104015 (2016).

[63] T. Damour, High-energy gravitational scattering and the general relativistic two-body problem, Phys. Rev. D 97, 044038 (2018).

[64] T. Damour, Classical and quantum scattering in postMinkowskian gravity, Phys. Rev. D 102, 024060 (2020).

[65] L. Blanchet and A. S. Fokas, Equations of motion of selfgravitating $N$-body systems in the first post-Minkowskian approximation, Phys. Rev. D 98, 084005 (2018).

[66] N. Arkani-Hamed, T.-C. Huang, and Y.-t. Huang, Scattering amplitudes for all masses and spins, arXiv:1709.04891.

[67] N. J. Bjerrum-Bohr, P. H. Damgaard, G. Festuccia, L. Planté, and P. Vanhove, General Relativity from Scattering Amplitudes, Phys. Rev. Lett. 121, 171601 (2018).

[68] D. A. Kosower, B. Maybee, and D. O'Connell, Amplitudes, observables, and classical scattering, J. High Energy Phys. 02 (2019) 137.

[69] C. Cheung, I. Z. Rothstein, and M. P. Solon, From Scattering Amplitudes to Classical Potentials in the Post-Minkowskian Expansion, Phys. Rev. Lett. 121, 251101 (2018).

[70] Y. F. Bautista and A. Guevara, From scattering amplitudes to classical physics: Universality, double copy and soft theorems, arXiv:1903.12419.

[71] Z. Bern, C. Cheung, R. Roiban, C.-H. Shen, M. P. Solon, and M. Zeng, Scattering Amplitudes and the Conservative Hamiltonian for Binary Systems at Third Post-Minkowskian Order, Phys. Rev. Lett. 122, 201603 (2019).

[72] Z. Bern, J. Parra-Martinez, R. Roiban, M. S. Ruf, C.-H. Shen, M. P. Solon, and M. Zeng, Scattering Amplitudes and Conservative Binary Dynamics at $\mathcal{O}\left(G^{4}\right)$, Phys. Rev. Lett. 126, 171601 (2021).

[73] N. E. J. Bjerrum-Bohr, A. Cristofoli, and P. H. Damgaard, Post-Minkowskian scattering angle in Einstein gravity, J. High Energy Phys. 08 (2020) 038.

[74] N. E. J. Bjerrum-Bohr, P. H. Damgaard, L. Planté, and P. Vanhove, The amplitude for classical gravitational scattering at third post-Minkowskian order, arXiv:2105.05218.

[75] A. Cristofoli, P. H. Damgaard, P. Di Vecchia, and C. Heissenberg, Second-order post-Minkowskian scattering in arbitrary dimensions, J. High Energy Phys. 07 (2020) 122.

[76] S. Foffa, Gravitating binaries at 5PN in the post-Minkowskian approximation, Phys. Rev. D 89, 024019 (2014).

[77] G. Kälin and R. A. Porto, From boundary data to bound states, J. High Energy Phys. 01 (2020) 072.

[78] G. Kälin, Z. Liu, and R. A. Porto, Conservative Dynamics of Binary Systems to Third Post-Minkowskian Order from the Effective Field Theory Approach, Phys. Rev. Lett. 125, 261103 (2020).

[79] G. Kälin and R. A. Porto, Post-Minkowskian effective field theory for conservative binary dynamics, J. High Energy Phys. 11 (2020) 106.

[80] C. Dlapa, G. Kälin, Z. Liu, and R. A. Porto, Dynamics of binary systems to fourth post-Minkowskian order from the effective field theory approach, arXiv:2106.08276.

[81] G. Mogull, J. Plefka, and J. Steinhoff, Classical black hole scattering from a worldline quantum field theory, J. High Energy Phys. 02 (2021) 048.
[82] G. U. Jakobsen, G. Mogull, J. Plefka, and J. Steinhoff, Classical Gravitational Bremsstrahlung from a Worldline Quantum Field Theory, Phys. Rev. Lett. 126, 201103 (2021).

[83] D. Bini and T. Damour, Gravitational spin-orbit coupling in binary systems, post-Minkowskian approximation and effective one-body theory, Phys. Rev. D 96, 104038 (2017).

[84] D. Bini and T. Damour, Gravitational spin-orbit coupling in binary systems at the second post-Minkowskian approximation, Phys. Rev. D 98, 044036 (2018).

[85] J. Vines, Scattering of two spinning black holes in postMinkowskian gravity, to all orders in spin, and effectiveone-body mappings, Classical Quantum Gravity 35, 084002 (2018).

[86] J. Vines, J. Steinhoff, and A. Buonanno, Spinningblack-hole scattering and the test-black-hole limit at second post-Minkowskian order, Phys. Rev. D 99, 064054 (2019).

[87] A. Guevara, A. Ochirov, and J. Vines, Black-hole scattering with general spin directions from minimal-coupling amplitudes, Phys. Rev. D 100, 104024 (2019).

[88] G. Kälin and R. A. Porto, From boundary data to bound states II: Scattering angle to dynamical invariants (with twist), J. High Energy Phys. 02 (2020) 120.

[89] Z. Bern, A. Luna, R. Roiban, C.-H. Shen, and M. Zeng, Spinning black hole binary dynamics, scattering amplitudes, and effective field theory, Phys. Rev. D 104, 065014 (2021).

[90] M.-Z. Chung, Y.-t. Huang, J.-W. Kim, and S. Lee, Complete Hamiltonian for spinning binary systems at first post-Minkowskian order, J. High Energy Phys. 05 (2020) 105.

[91] B. Maybee, D. O'Connell, and J. Vines, Observables and amplitudes for spinning particles and black holes, J. High Energy Phys. 12 (2019) 156.

[92] Y. F. Bautista, A. Guevara, C. Kavanagh, and J. Vines, From scattering in black hole backgrounds to higher-spin amplitudes: Part I, arXiv:2107.10179.

[93] D. Kosmopoulos and A. Luna, Quadratic-in-spin Hamiltonian at $\mathcal{O}\left(\mathrm{G}^{2}\right)$ from scattering amplitudes, J. High Energy Phys. 07 (2021) 037.

[94] Z. Liu, R. A. Porto, and Z. Yang, Spin effects in the effective field theory approach to post-Minkowskian conservative dynamics, J. High Energy Phys. 06 (2021) 012.

[95] G. U. Jakobsen, G. Mogull, J. Plefka, and J. Steinhoff, Gravitational Bremsstrahlung and hidden supersymmetry of spinning bodies, arXiv:2106.10256.

[96] G. U. Jakobsen, G. Mogull, J. Plefka, and J. Steinhoff, SUSY in the sky with gravitons, arXiv:2109.04465.

[97] T. Damour, Radiative contribution to classical gravitational scattering at the third order in $G$, Phys. Rev. D 102, 124008 (2020).

[98] D. Bini, T. Damour, and A. Geralico, Radiative contributions to gravitational scattering, Phys. Rev. D 104, 084031 (2021).

[99] D. Bini and A. Geralico, Higher-order tail contributions to the energy and angular momentum fluxes in a two-body scattering process, Phys. Rev. D 104, 104020 (2021). 
[100] D. Bini and A. Geralico, Frequency domain analysis of the gravitational wave energy loss in hyperbolic encounters, Phys. Rev. D 104, 104019 (2021).

[101] M. V. S. Saketh, J. Vines, J. Steinhoff, and A. Buonanno, Conservative and radiative dynamics in classical relativistic scattering and bound systems, arXiv:2109.05994.

[102] Y. Mino, M. Sasaki, and T. Tanaka, Gravitational radiation reaction to a particle motion, Phys. Rev. D 55, 3457 (1997).

[103] T. C. Quinn and R. M. Wald, An axiomatic approach to electromagnetic and gravitational radiation reaction of particles in curved spacetime, Phys. Rev. D 56, 3381 (1997).

[104] L. Barack, Y. Mino, H. Nakano, A. Ori, and M. Sasaki, Calculating the Gravitational Self-Force in Schwarzschild Spacetime, Phys. Rev. Lett. 88, 091101 (2002).

[105] L. Barack and A. Ori, Gravitational Self-Force on a Particle Orbiting a Kerr Black Hole, Phys. Rev. Lett. 90, 111101 (2003).

[106] S. L. Detweiler and B. F. Whiting, Self-force via a Green's function decomposition, Phys. Rev. D 67, 024025 (2003).

[107] L. Barack and A. Ori, Regularization parameters for the selfforce in Schwarzschild spacetime. 2. Gravitational and electromagnetic cases, Phys. Rev. D 67, 024029 (2003).

[108] S. L. Detweiler, Perspective on gravitational self-force analyses, Classical Quantum Gravity 22, S681 (2005).

[109] E. Rosenthal, Second-order gravitational self-force, Phys. Rev. D 74, 084018 (2006).

[110] T. Hinderer and E. E. Flanagan, Two timescale analysis of extreme mass ratio inspirals in Kerr. I. Orbital motion, Phys. Rev. D 78, 064028 (2008).

[111] S. E. Gralla and R. M. Wald, A rigorous derivation of gravitational self-force, Classical Quantum Gravity 25, 205009 (2008); 28, 159501(E) (2011).

[112] A. G. Shah, T. S. Keidl, J. L. Friedman, D.-H. Kim, and L. R. Price, Conservative, gravitational self-force for a particle in circular orbit around a Schwarzschild black hole in a radiation gauge, Phys. Rev. D 83, 064018 (2011).

[113] T. S. Keidl, A. G. Shah, J. L. Friedman, D.-H. Kim, and L. R. Price, Gravitational self-force in a radiation gauge, Phys. Rev. D 82, 124012 (2010); 90, 109902(E) (2014).

[114] A. Pound, Singular perturbation techniques in the gravitational self-force problem, Phys. Rev. D 81, 124009 (2010).

[115] L. Barack and N. Sago, Beyond the geodesic approximation: Conservative effects of the gravitational self-force in eccentric orbits around a Schwarzschild black hole, Phys. Rev. D 83, 084023 (2011).

[116] A. Pound, Second-Order Gravitational Self-Force, Phys. Rev. Lett. 109, 051101 (2012).

[117] A. Pound, Nonlinear gravitational self-force. I. Field outside a small body, Phys. Rev. D 86, 084019 (2012).

[118] S. E. Gralla, Second-order gravitational self-force, Phys. Rev. D 85, 124011 (2012).

[119] A. Pound, Conservative effect of the second-order gravitational self-force on quasicircular orbits in Schwarzschild spacetime, Phys. Rev. D 90, 084039 (2014).

[120] A. Pound, Nonlinear gravitational self-force: Second-order equation of motion, Phys. Rev. D 95, 104056 (2017).
[121] M. van de Meent, Gravitational self-force on eccentric equatorial orbits around a Kerr black hole, Phys. Rev. D 94, 044034 (2016).

[122] M. van de Meent, Gravitational self-force on generic bound geodesics in Kerr spacetime, Phys. Rev. D 97, 104033 (2018).

[123] L. Barack, Gravitational self-force in extreme mass-ratio inspirals, Classical Quantum Gravity 26, 213001 (2009).

[124] A. Pound, Motion of small objects in curved spacetimes: An introduction to gravitational self-force, Fund. Theor. Phys. 179, 399 (2015).

[125] L. Barack and A. Pound, Self-force and radiation reaction in general relativity, Rep. Prog. Phys. 82, 016904 (2019).

[126] A. Pound and B. Wardell, Black hole perturbation theory and gravitational self-force, arXiv:2101.04592.

[127] S. L. Detweiler, A consequence of the gravitational selfforce for circular orbits of the Schwarzschild geometry, Phys. Rev. D 77, 124026 (2008).

[128] D. Bini and T. Damour, High-order post-Newtonian contributions to the two-body gravitational interaction potential from analytical gravitational self-force calculations, Phys. Rev. D 89, 064063 (2014).

[129] C. Kavanagh, A. C. Ottewill, and B. Wardell, Analytical high-order post-Newtonian expansions for extreme mass ratio binaries, Phys. Rev. D 92, 084025 (2015).

[130] S. Hopper, C. Kavanagh, and A. C. Ottewill, Analytic selfforce calculations in the post-Newtonian regime: Eccentric orbits on a Schwarzschild background, Phys. Rev. D 93, 044010 (2016).

[131] D. Bini, T. Damour, and A. Geralico, Confirming and improving post-Newtonian and effective-one-body results from self-force computations along eccentric orbits around a Schwarzschild black hole, Phys. Rev. D 93, 064023 (2016).

[132] C. Kavanagh, A. C. Ottewill, and B. Wardell, Analytical high-order post-Newtonian expansions for spinning extreme mass ratio binaries, Phys. Rev. D 93, 124038 (2016).

[133] D. Bini, T. Damour, A. Geralico, and C. Kavanagh, Detweiler's redshift invariant for spinning particles along circular orbits on a Schwarzschild background, Phys. Rev. D 97, 104022 (2018).

[134] D. Bini and A. Geralico, New gravitational self-force analytical results for eccentric equatorial orbits around a Kerr black hole: Redshift invariant, Phys. Rev. D 100, 104002 (2019).

[135] D. Bini, A. Geralico, and J. Steinhoff, Detweiler's redshift invariant for extended bodies orbiting a Schwarzschild black hole, Phys. Rev. D 102, 024091 (2020).

[136] S. R. Dolan, N. Warburton, A. I. Harte, A. Le Tiec, B. Wardell, and L. Barack, Gravitational self-torque and spin precession in compact binaries, Phys. Rev. D 89, 064011 (2014).

[137] D. Bini and T. Damour, Two-body gravitational spin-orbit interaction at linear order in the mass ratio, Phys. Rev. D 90, 024039 (2014).

[138] S. Akcay, D. Dempsey, and S. R. Dolan, Spin-orbit precession for eccentric black hole binaries at first order in the mass ratio, Classical Quantum Gravity 34, 084001 (2017). 
[139] C. Kavanagh, D. Bini, T. Damour, S. Hopper, A. C. Ottewill, and B. Wardell, Spin-orbit precession along eccentric orbits for extreme mass ratio black hole binaries and its effective-one-body transcription, Phys. Rev. D 96, 064012 (2017).

[140] D. Bini, T. Damour, A. Geralico, C. Kavanagh, and M. van de Meent, Gravitational self-force corrections to gyroscope precession along circular orbits in the Kerr spacetime, Phys. Rev. D 98, 104062 (2018).

[141] D. Bini and A. Geralico, New gravitational self-force analytical results for eccentric equatorial orbits around a Kerr black hole: Gyroscope precession, Phys. Rev. D 100, 104003 (2019).

[142] A. Pound, B. Wardell, N. Warburton, and J. Miller, Second-Order Self-Force Calculation of the Gravitational Binding Energy in Compact Binaries, Phys. Rev. Lett. 124, 021101 (2020).

[143] N. Warburton, A. Pound, B. Wardell, J. Miller, and L. Durkan, Gravitational-Wave Energy Flux for Compact Binaries through Second Order in the Mass Ratio, Phys. Rev. Lett. 127, 151102 (2021).

[144] A. Buonanno and T. Damour, Effective one-body approach to general relativistic two-body dynamics, Phys. Rev. D 59, 084006 (1999).

[145] A. Buonanno and T. Damour, Transition from inspiral to plunge in binary black hole coalescences, Phys. Rev. D 62, 064015 (2000).

[146] T. Damour, P. Jaranowski, and G. Schäfer, On the determination of the last stable orbit for circular general relativistic binaries at the third post-Newtonian approximation, Phys. Rev. D 62, 084011 (2000).

[147] A. Buonanno, Y. Pan, J. G. Baker, J. Centrella, B. J. Kelly, S. T. McWilliams, and J. R. van Meter, Toward faithful templates for nonspinning binary black holes using the effective-one-body approach, Phys. Rev. D 76, 104049 (2007).

[148] T. Damour, B. R. Iyer, and A. Nagar, Improved resummation of post-Newtonian multipolar waveforms from circularized compact binaries, Phys. Rev. D 79, 064004 (2009).

[149] Y. Pan, A. Buonanno, M. Boyle, L. T. Buchman, L. E. Kidder, H. P. Pfeiffer, and M. A. Scheel, Inspiralmerger-ringdown multipolar waveforms of nonspinning black-hole binaries using the effective-one-body formalism, Phys. Rev. D 84, 124052 (2011).

[150] A. Nagar, G. Pratten, G. Riemenschneider, and R. Gamba, Multipolar effective one body model for nonspinning black hole binaries, Phys. Rev. D 101, 024041 (2020).

[151] T. Damour, P. Jaranowski, and G. Schäfer, Effective one body approach to the dynamics of two spinning black holes with next-to-leading order spin-orbit coupling, Phys. Rev. D 78, 024009 (2008).

[152] E. Barausse and A. Buonanno, An improved effective-onebody Hamiltonian for spinning black-hole binaries, Phys. Rev. D 81, 084024 (2010).

[153] E. Barausse and A. Buonanno, Extending the effectiveone-body Hamiltonian of black-hole binaries to include next-to-next-to-leading spin-orbit couplings, Phys. Rev. D 84, 104027 (2011).

[154] A. Nagar, Effective one-body Hamiltonian of two spinning black-holes with next-to-next-to-leading order spin-orbit coupling, Phys. Rev. D 84, 084028 (2011); 88, 089901(E) (2013).

[155] T. Damour and A. Nagar, New effective-one-body description of coalescing nonprecessing spinning black-hole binaries, Phys. Rev. D 90, 044018 (2014).

[156] S. Balmelli and T. Damour, New effective-one-body Hamiltonian with next-to-leading order spin-spin coupling, Phys. Rev. D 92, 124022 (2015).

[157] M. Khalil, J. Steinhoff, J. Vines, and A. Buonanno, Fourth post-Newtonian effective-one-body Hamiltonians with generic spins, Phys. Rev. D 101, 104034 (2020).

[158] Y. Pan, A. Buonanno, A. Taracchini, L. E. Kidder, A. H. Mroué, H. P. Pfeiffer, M. A. Scheel, and B. Szilágyi, Inspiral-merger-ringdown waveforms of spinning, precessing black-hole binaries in the effective-one-body formalism, Phys. Rev. D 89, 084006 (2014).

[159] A. Taracchini, Y. Pan, A. Buonanno, E. Barausse, M. Boyle, T. Chu, G. Lovelace, H. P. Pfeiffer, and M. A. Scheel, Prototype effective-one-body model for nonprecessing spinning inspiral-merger-ringdown waveforms, Phys. Rev. D 86, 024011 (2012).

[160] A. Taracchini et al., Effective-one-body model for blackhole binaries with generic mass ratios and spins, Phys. Rev. D 89, 061502 (2014).

[161] S. Babak, A. Taracchini, and A. Buonanno, Validating the effective-one-body model of spinning, precessing binary black holes against numerical relativity, Phys. Rev. D 95, 024010 (2017).

[162] A. Bohé et al., Improved effective-one-body model of spinning, nonprecessing binary black holes for the era of gravitational-wave astrophysics with advanced detectors, Phys. Rev. D 95, 044028 (2017).

[163] R. Cotesta, A. Buonanno, A. Bohé, A. Taracchini, I. Hinder, and S. Ossokine, Enriching the symphony of gravitational waves from binary black holes by tuning higher harmonics, Phys. Rev. D 98, 084028 (2018).

[164] S. Ossokine et al., Multipolar effective-one-body waveforms for precessing binary black holes: Construction and validation, Phys. Rev. D 102, 044055 (2020).

[165] A. Nagar, F. Messina, P. Rettegno, D. Bini, T. Damour, A. Geralico, S. Akcay, and S. Bernuzzi, Nonlinear-in-spin effects in effective-one-body waveform models of spinaligned, inspiralling, neutron star binaries, Phys. Rev. D 99, 044007 (2019).

[166] A. Nagar et al., Time-domain effective-one-body gravitational waveforms for coalescing compact binaries with nonprecessing spins, tides and self-spin effects, Phys. Rev. D 98, 104052 (2018).

[167] D. Bini and T. Damour, Gravitational radiation reaction along general orbits in the effective one-body formalism, Phys. Rev. D 86, 124012 (2012).

[168] T. Hinderer and S. Babak, Foundations of an effective-onebody model for coalescing binaries on eccentric orbits, Phys. Rev. D 96, 104048 (2017).

[169] A. Nagar, A. Bonino, and P. Rettegno, Effective one-body multipolar waveform model for spin-aligned, quasicircular, eccentric, hyperbolic black hole binaries, Phys. Rev. D 103, 104021 (2021).

[170] M. Khalil, A. Buonanno, J. Steinhoff, and J. Vines, Radiation-reaction force and multipolar waveforms for 
eccentric, spin-aligned binaries in the effective-one-body formalism, Phys. Rev. D 104, 024046 (2021).

[171] A. Antonelli, A. Buonanno, J. Steinhoff, M. van de Meent, and J. Vines, Energetics of two-body Hamiltonians in post-Minkowskian gravity, Phys. Rev. D 99, 104004 (2019).

[172] P. H. Damgaard and P. Vanhove, Remodeling the effective one-body formalism in post-Minkowskian gravity, Phys. Rev. D 104, 104029 (2021).

[173] T. Damour, Gravitational self-force in a Schwarzschild background and the effective one-body formalism, Phys. Rev. D 81, 024017 (2010).

[174] E. Barausse, A. Buonanno, and A. Le Tiec, Complete nonspinning effective-one-body metric at linear order in the mass ratio, Phys. Rev. D 85, 064010 (2012).

[175] S. Akcay, L. Barack, T. Damour, and N. Sago, Gravitational self-force and the effective-one-body formalism between the innermost stable circular orbit and the light ring, Phys. Rev. D 86, 104041 (2012).

[176] A. Antonelli, M. van de Meent, A. Buonanno, J. Steinhoff, and J. Vines, Quasicircular inspirals and plunges from nonspinning effective-one-body Hamiltonians with gravitational self-force information, Phys. Rev. D 101, 024024 (2020).

[177] D. Bini, T. Damour, and A. Geralico, Novel Approach to Binary Dynamics: Application to the Fifth Post-Newtonian Level, Phys. Rev. Lett. 123, 231104 (2019).

[178] D. Bini, T. Damour, and A. Geralico, Binary dynamics at the fifth and fifth-and-a-half post-Newtonian orders, Phys. Rev. D 102, 024062 (2020).

[179] D. Bini, T. Damour, and A. Geralico, Sixth postNewtonian nonlocal-in-time dynamics of binary systems, Phys. Rev. D 102, 084047 (2020).

[180] D. Bini, T. Damour, and A. Geralico, Sixth postNewtonian local-in-time dynamics of binary systems, Phys. Rev. D 102, 024061 (2020).

[181] A. Antonelli, C. Kavanagh, M. Khalil, J. Steinhoff, and J. Vines, Gravitational Spin-Orbit ccoupling through ThirdSubleading Post-Newtonian Order: From First-Order Self-Force to Arbitrary Mass Ratios, Phys. Rev. Lett. 125, 011103 (2020).

[182] A. Antonelli, C. Kavanagh, M. Khalil, J. Steinhoff, and J. Vines, Gravitational spin-orbit and aligned $\operatorname{spin}_{1}-\mathrm{spin}_{2}$ couplings through third-subleading post-Newtonian orders, Phys. Rev. D 102, 124024 (2020).

[183] See Supplemental Material at http://link.aps.org/ supplemental/10.1103/PhysRevD.104.124015 for the complete expressions of the results of this paper. The file SO_5.5PN.m in the Supplemental Material contains expressions for the 5.5PN spin-orbit Hamiltonian, scattering angle, redshift and spin-precession invariants, radial action, and circular-orbit binding energy.

[184] L. Blanchet and T. Damour, Tail transported temporal correlations in the dynamics of a gravitating system, Phys. Rev. D 37, 1410 (1988).

[185] L. Bernard, L. Blanchet, A. Bohé, G. Faye, and S. Marsat, Fokker action of nonspinning compact binaries at the fourth post-Newtonian approximation, Phys. Rev. D 93, 084037 (2016).
[186] M. H. L. Pryce, The mass-centre in the restricted theory of relativity and its connexion with the quantum theory of elementary particles, Proc. R. Soc. A 195, 62 (1948).

[187] T.D. Newton and E. P. Wigner, Localized states for elementary systems, Rev. Mod. Phys. 21, 400 (1949).

[188] L. Blanchet and T. Damour, Post-newtonian generation of gravitational waves, in Annales de l'IHP Physique théorique (Institut Henri Poincaré, Paris, 1989), Vol. 50, pp. 377-408.

[189] L. E. Kidder, Coalescing binary systems of compact objects to post-Newtonian 5/2 order. 5. Spin effects, Phys. Rev. D 52, 821 (1995).

[190] T. Damour and N. Deruelle, General relativistic celestial mechanics of binary systems. I. The post-newtonian motion, in Annales de l'IHP Physique théorique (Institut Henri Poincaré, Paris, 1985), Vol. 43, pp. 107-132.

[191] R.-M. Memmesheimer, A. Gopakumar, and G. Schaefer, Third post-Newtonian accurate generalized quasiKeplerian parametrization for compact binaries in eccentric orbits, Phys. Rev. D 70, 104011 (2004).

[192] M. Tessmer, J. Hartung, and G. Schafer, Motion and gravitational wave forms of eccentric compact binaries with orbital-angular-momentum-aligned spins under nextto-leading order in spin-orbit and leading order in spin(1)spin(2) and spin-squared couplings, Classical Quantum Gravity 27, 165005 (2010).

[193] M. Tessmer, J. Hartung, and G. Schafer, Aligned spins: Orbital elements, decaying orbits, and last stable circular orbit to high post-Newtonian orders, Classical Quantum Gravity 30, 015007 (2013).

[194] C. M. Will, Post-Newtonian gravitational radiation and equations of motion via direct integration of the relaxed Einstein equations. III. Radiation reaction for binary systems with spinning bodies, Phys. Rev. D 71, 084027 (2005).

[195] W. Tulczyjew, Equations of motion of rotating bodies in general relativity theory, Acta Phys. Polon. 18, 37 (1959); 18, 534(E) (1959).

[196] W. G. Dixon, Extended bodies in general relativity: Their description and motion, in Isolated Gravitating Systems in General Relativity, edited by J. Ehlers (North Holland, Amsterdam, 1979).

[197] J. Vines, D. Kunst, J. Steinhoff, and T. Hinderer, Canonical Hamiltonian for an extended test body in curved spacetime: To quadratic order in spin, Phys. Rev. D 93, 103008 (2016).

[198] D. Bini, A. Geralico, and J. Vines, Hyperbolic scattering of spinning particles by a Kerr black hole, Phys. Rev. D 96, 084044 (2017).

[199] A. Le Tiec, L. Blanchet, and B. F. Whiting, The first law of binary black hole mechanics in general relativity and postNewtonian theory, Phys. Rev. D 85, 064039 (2012).

[200] L. Blanchet, A. Buonanno, and A. Le Tiec, First law of mechanics for black hole binaries with spins, Phys. Rev. D 87, 024030 (2013).

[201] A. Le Tiec, First law of mechanics for compact binaries on eccentric orbits, Phys. Rev. D 92, 084021 (2015).

[202] L. Blanchet and A. Le Tiec, First law of compact binary mechanics with gravitational-wave tails, Classical Quantum Gravity 34, 164001 (2017). 
[203] D. Bini, T. Damour, and A. Geralico, High post-Newtonian order gravitational self-force analytical results for eccentric equatorial orbits around a Kerr black hole, Phys. Rev. D 93, 124058 (2016).

[204] Black Hole Perturbation Toolkit, http://bhptoolkit.org/.

[205] T. Damour, A. Nagar, D. Pollney, and C. Reisswig, Energy versus Angular Momentum in Black Hole Binaries, Phys. Rev. Lett. 108, 131101 (2012).

[206] A. Nagar, T. Damour, C. Reisswig, and D. Pollney, Energetics and phasing of nonprecessing spinning coalescing black hole binaries, Phys. Rev. D 93, 044046 (2016).

[207] M. Ruiz, R. Takahashi, M. Alcubierre, and D. Nunez, Multipole expansions for energy and momenta carried by gravitational waves, Gen. Relativ. Gravit. 40, 2467 (2008).

[208] T. Dietrich, S. Bernuzzi, M. Ujevic, and W. Tichy, Gravitational waves and mass ejecta from binary neutron star mergers: Effect of the stars' rotation, Phys. Rev. D 95, 044045 (2017).

[209] S. Ossokine, T. Dietrich, E. Foley, R. Katebi, and G. Lovelace, Assessing the energetics of spinning binary black hole systems, Phys. Rev. D 98, 104057 (2018).

[210] https://data.black-holes.org/waveforms.

[211] M. Boyle et al., The SXS Collaboration catalog of binary black hole simulations, Classical Quantum Gravity 36, 195006 (2019).

[212] D. Bini and T. Damour, Gravitational scattering of two black holes at the fourth post-Newtonian approximation, Phys. Rev. D 96, 064021 (2017).

[213] D. Bini, T. Damour, A. Geralico, S. Laporta, and P. Mastrolia, Gravitational dynamics at $O\left(G^{6}\right)$ : Perturbative gravitational scattering meets experimental mathematics, arXiv:2008.09389.

[214] D. Bini, T. Damour, A. Geralico, S. Laporta, and P. Mastrolia, Gravitational scattering at the seventh order in $G$ : Nonlocal contribution at the sixth post-Newtonian accuracy, Phys. Rev. D 103, 044038 (2021).

[215] G. Cho, A. Gopakumar, M. Haney, and H. M. Lee, Gravitational waves from compact binaries in
post-Newtonian accurate hyperbolic orbits, Phys. Rev. D 98, 024039 (2018).

[216] B. P. Abbott et al. (KAGRA, LIGO Scientific, Virgo, and VIRGO Collaborations), Prospects for observing and localizing gravitational-wave transients with Advanced LIGO, Advanced Virgo and KAGRA, Living Rev. Relativity 21, 3 (2018).

[217] P. Amaro-Seoane et al. (LISA Collaboration), Laser interferometer space antenna, arXiv:1702.00786.

[218] M. Punturo et al., The Einstein Telescope: A thirdgeneration gravitational wave observatory, Classical Quantum Gravity 27, 194002 (2010).

[219] S. Kawamura et al., The Japanese space gravitational wave antenna DECIGO, Classical Quantum Gravity 23, S125 (2006).

[220] D. Reitze et al., Cosmic explorer: The U.S. contribution to gravitational-wave astronomy beyond LIGO, Bull. Am. Astron. Soc. 51, 035 (2019).

[221] M. Hannam, P. Schmidt, A. Bohé, L. Haegel, S. Husa, F. Ohme, G. Pratten, and M. Pürrer, Simple Model of Complete Precessing Black-Hole-Binary Gravitational Waveforms, Phys. Rev. Lett. 113, 151101 (2014).

[222] S. Husa, S. Khan, M. Hannam, M. Pürrer, F. Ohme, X. Jiménez Forteza, and A. Bohé, Frequency-domain gravitational waves from nonprecessing black-hole binaries. I. New numerical waveforms and anatomy of the signal, Phys. Rev. D 93, 044006 (2016).

[223] S. Khan, F. Ohme, K. Chatziioannou, and M. Hannam, Including higher order multipoles in gravitational-wave models for precessing binary black holes, Phys. Rev. D 101, 024056 (2020).

[224] G. Pratten, S. Husa, C. Garcia-Quiros, M. Colleoni, A. Ramos-Buades, H. Estelles, and R. Jaume, Setting the cornerstone for a family of models for gravitational waves from compact binaries: The dominant harmonic for nonprecessing quasicircular black holes, Phys. Rev. D 102, 064001 (2020). 